The Ages of the Stars

Y. Lebreton, D. Valls-Gabaud \& C. Charbonnel (eds)

EAS Publications Series, Vol. 65, 2014

\title{
BAYESIAN ISOCHRONE FITTING AND STELLAR AGES
}

\author{
David Valls-Gabaud ${ }^{1}$
}

\begin{abstract}
Stellar evolution theory has been extraordinarily successful at explaining the different phases under which stars form, evolve and die. While the strongest constraints have traditionally come from binary stars, the advent of asteroseismology is bringing unique measures in well-characterised stars. For stellar populations in general, however, only photometric measures are usually available, and the comparison with the predictions of stellar evolution theory have mostly been qualitative. For instance, the geometrical shapes of isochrones have been used to infer ages of coeval populations, but without any proper statistical basis. In this chapter we provide a pedagogical review on a Bayesian formalism to make quantitative inferences on the properties of single, binary and small ensembles of stars, including unresolved populations. As an example, we show how stellar evolution theory can be used in a rigorous way as a prior information to measure the ages of stars between the ZAMS and the Helium flash, and their uncertainties, using photometric data only.
\end{abstract}

\section{Introduction}

In this chapter a brief summary is presented of the uses of stellar evolution theory to infer properties of single stars (Section 3), of detached binary stars whose components are assumed to have evolved independently of each other (Section 4), and coeval stellar populations such as (presumably) those in clusters (Section 5). When the fundamental properties of the star(s) in question are known (mass, absolute luminosity, effective temperature, etc), stellar tracks computed for this particular (set of) star(s) can be used to infer further properties, such as ages. In general, however, one wishes to use the predictions of stellar evolution to infer these properties. The data at hand are usually magnitudes and colours, hence

1 LERMA, CNRS UMR 8112, Observatoire de Paris, 61 Avenue de l'Observatoire, 75014

Paris, France. Email david.valls-gabaud@obspm.fr

(c) EDP Sciences 2016

DOI: (will be inserted later) 
The Ages of the Stars

the interpretation of the features in the colour-magnitude diagrammes (CMDs) is carried out with isochrones rather than stellar tracks. The thorny issue of transforming isochrones from/to the theoretical diagram to/from observed CMDs will not be dealt with here (see the contributions by Cassisi, and by Lebreton, Goupil and Montalbán in this volume), and constitute one of the sources of systematic uncertainties. One also has to bear in mind that while many efforts have been placed to find the best transformations, the differences observed cannot (yet?) be fully ascribed to either systematics in the observations or in missing/wrong physics in the stellar evolutionary calculations. For instance the recent analysis by VandenBerg et al. (2010) for some globular clusters, and by An et al. (2007) for open clusters show that while some CMDs can be well fitted, other colour-magnitude combinations of the same clusters show anomalies which go well beyond the corrections for systematics, rotation, activity, transformations, metallicity, etc. Empirical bolometric corrections are another source of uncertainty (Torres, 2010) as are the systematics in the determination of effective temperatures (e.g., Ramírez \& Meléndez, 2005). Likewise, it does make sense to adopt a standard set of values (Harmanec \& Prša, 2011) with nominal values to avoid some of the systematics arising with the adoption of different key values (solar radius, mass, etc). Section 6 deals with the general problem of inverting the CMDs of a mixture of resolved stellar populations to infer their distribution of ages and hence their chemical and star formation rate histories, while Section 7 is a very brief discussion on CMDs of pixels in unresolved stellar populations. Section 8 closes this chapter with a discussion on some statistical issues in the interpretation of CMDs. We will limit the scope of this chapter to stars from the main sequence to the Helium flash, as the predictions in this range appear to be the most robust ones. The white dwarf phase can also be used in a rather robust way (provided the cooling and the equation of state are properly characterised), as described by T. von Hippel in this volume.

\section{Colour-Magnitude and Hertzsprung-Russell Diagrammes}

The plotting of the colors (or spectra) of stars as abscissae against their absolute magnitudes (total magnitudes) has become one of the most lucrative adventures in the study of star light.

Shapley (1960)

It is appropriate to recall, in the context of this volume, that just over a century ago the first colour-magnitude diagram (CMD) was published. The author of this landmark paper was not Ejnar Hertzsprung nor Henry N. Russell, but Hans O. Rosenberg, a colleague of Karl Schwarzschild at Göttingen. Rosenberg had been working since 1907 on getting spectral properties of stars by measuring plates obtained with the Zeiss objective prism camera (Hermann, 1994). To maximise the number of spectra per plate, he observed the Pleiades cluster and obtained spectra 
for about 60 of them, over 1907-1909, noting that their inferred effective temperatures correlated with their apparent magnitudes in the first ever published CMD (Rosenberg, 1910) ${ }^{1}$. His goal was to "make the most accurate determination of the spectral types of stars in the Pleiades" by using a "physiological blend" of the depth and width of the Ca II K line $(393.37 \mathrm{~nm})$ with the Balmer $\mathrm{H} \delta$ and $\mathrm{H} \zeta$ lines. $\mathrm{He}$ excluded the $\mathrm{Ca}$ II $\mathrm{H}$ line at $396.9 \mathrm{~nm}$ as it was blended with $\mathrm{H} \epsilon$ in the very low dispersion spectra he used $(1.9 \mathrm{~mm}$ from $\mathrm{H} \gamma$ to $\mathrm{H} \zeta)$. With an exposure time of 90 minutes he could measure spectra down to the 10th photographic magnitude, finding that for the actual members of the Pleiades "there is a strict relation between the brightness and the spectral type, with no exception in the interval from the 3rd to the 9th magnitude.". Hertzsprung's diagrams (magnitude vs colour) of the Pleiades and the Hyades would appear a year later (Hertzsprung, 1911) while Russell's version for field stars with parallaxes (with absolute magnitude vs spectral type) would only appear in 1914 (Russell, 1914a,b), although the correlation between luminosity and spectral type was noted by Hertzsprung in 1905 and in more detail by Russell (1912). The key difference is that while Russell required parallaxes to ascertain the distances to the stars, Rosenberg carefully checked the membership of stars in the Pleiades, rejecting outliers and non-members.

Henry Russell was obsessed by priority, (self-)attribution and promotion (DeVorkin, 2000). For example, the famous Vogt(-Russell) theorem on stellar structure first appeared in 1926 (Vogt, 1926) and in his influential textbook Russell does give full credit to Vogt (Russell, Dugan \& Stewart, 1927), yet he will later claim (Russell, 1931) that he had found it independently. In the case of the CMD, Russell called it in private the "Russell diagram", but this was not accepted in public, as the contribution by Hertzsprung (unlike Rosenberg's) was well and widely known. Russell poured over the astronomical journals, and was well aware of Hertzsprung's results. We know he read the Astronomischen Nachrichten systematically, as one of the leading journals of the time, and that we was well aware of Hertzsprung's papers just as was his mentor, E.C. Pickering, who received them and wrote to Hertzsprung discussing several issues in spectral classification. Russell wrote to Hertzsprung on September 27, 1910, thanking him for sending copies of his papers (Hearnshaw, 1986). Hertzsprung (1911)'s paper contained the CMDs of the Hyades and the Pleiades, citing explicitely the previous -and pioneeringwork by Rosenberg (1910). With the raising influence of European (mosly Dutch) astronomers in the USA, the issue of the proper acknowledgement became very serious and created frictions and debate within the community. After two decades, the "Russell diagram" became known as the Hertzsprung-Russell diagram, thanks in part to the influential conference delivered in 1933 by B. Strömgren at the meeting of the Astronomische Gesellschaft, but much to the irritation of many, including Russell himself who even refused to acknowledge that Hertzsprung had found (and coined the terms) 'giant' and 'dwarf' stars (Smith, 1977). The (proper) renaming of the diagram was a long battle which lasted till the late 1940s, when

\footnotetext{
${ }^{1}$ A translation into English is available at Leos Ondra's website www . leosondra.cz/en/firsthr-diagram
} 
The Ages of the Stars

S. Chandraskhar, advising the Astrophysical Journal and tired of the controversy, decided that the standard nomenclature would be the "H-R Diagram" (DeVorkin, 2000). Rosenberg's pioneering contribution has been unfairly forgotten from the history describing the elaboration of the first CMDs (see, e.g., Waterfield, 1956; Nielsen, 1969; DeVorkin, 2000).

Hans Rosenberg was born in Berlin on May 18, 1879, and studied first in Berlin, under W. Foerster, and then in Strasbourg, with E. Becker, obtaining his Dr. Phil. with a thesis on the period changes that $\chi$ Cygni underwent from 1686 to 1901. Interested in both instrumentation and astrophysics, he moved to Göttingen to work with Karl Schwarzschild where he was to produce the first large survey of stellar temperatures estimated with objetive prism spectra. For years, the Rosenberg temperature scale will set the standard and will be widely used, as well as his review on photoelectric photometry in the Handbuch der Astrophysik (Rosenberg, 1929). His Habilitation thesis from Tübingen in 1910 was on "The relation between brightness and spectral type in the Pleiades", whose results were published in the above-cited key paper in Astr. Nach. (Rosenberg, 1910). His instrumental expertise allowed him to get spectra of comets Daniel (1907 IV) and Morehouse (1908 III). From 1910 on, he worked at Tübingen first as Privatdozent, where he founded its observatory in Österberg, becoming its director in 1912 and professor at the university in 1916 while serving in the army during World War I. He made with P. Goetz the first photometric map of the Moon, and developed the use of photoelectric cells as astronomical detectors in 1913. Moving to Kiel in 1925 he worked on solar eclipses, developed direct measures of the colours of stars and had heavy teaching duties. In spite of his position as professor at Kiel university, on April 1, 1933, three uniformed members of the feared SA Nazi paramilitary group came to his appartment and forced him to resign (Duerbeck, 2006), but he would have lost his position all the same with the racial laws enacted in 1935. He was immediately invited in 1934 by the University of Chicago to work at Yerkes Observatory where he stayed three years, working on measures of the limb darkening and colour indices in eclipsing binaries (e.g., Rosenberg, 1936). In 1938 he was appointed director of Istanbul Observatory, where he reorganised the teaching of astronomy at the University of Istanbul and set up new priorities for the observing campaigns. He passed away there on July 26, 1940, a week after suffering a heat stroke (Gleissberg, 1940).

An even earlier relationship between the colour and the magnitude was found by Charlier (1889), who studied the correlation between magnitude and a colourlike quantity (the difference between the magnitude he measured in a photographic plate and the visual magnitude) as determined by Max Wolf in Heidelberg. However, he interpreted the correlation found (the visually fainter stars had larger differences) as a systematic error in the visual magnitudes. As Rosenberg correctly pointed out, this could also be produced by selective reddening, hence the importance of getting spectra. The spectral type - magnitude correlation found in the Pleiades could not be produced by dust, hence "the plausible colour differences among the stars in the Pleiades -the fainter the star, the redder it is-following from the optical and photographic brightness measurements are confirmed by the 
spectral properties". This ground-breaking result will be taken to good use to lay the foundations of stellar physics (see, e.g., Salaris \& Cassisi, 2005), but the true pioneer has unfairly been forgotten to the extent that it would be a fitting tribute to rename the diagram as the Rosenberg-Hertzsprung-Russell diagram (RHR). Ironically, HR also stands for Hans Rosenberg's initials.

The final word may come from Hertzsprung himself. His modesty made him to avoid talking about his own contributions to astronomy, and, as Strand (1968) reminds us, he remarked on the controverted issue of the naming of the diagram:

"Why not call it the colour-magnitude diagram? Then we know what it is all about."

The use of CMDs to constrain the physical properties of the stars was noticed very quickly. In fact, Russell (1912) was the very first to use the correlation between absolute magnitude and spectral type for (dwarf) stars with measured parallaxes to infer a distance to the Pleiades of 500 light-years ${ }^{2}$.

\section{Single stars}

The fitting of isochrones to a set of stars is the main method to constrain their physical properties, besides other techniques which are limited to nearby stars such as stellar oscillations. The set of basic properties includes the age $t$, Helium abundance $Y$, metallicity $Z, \alpha$ elements over abundance, distance modulus $\mu=m-M$, convection mixing length, etc, that is, all the quantities which determine the position of stars in a CMD. In contrast with its importance for stellar evolution, relatively little work has been done to formulate mathematically the problem to go beyond the 'fit-by-eye' approach which has characterised this field (and unfortunately still does!). It seems that the first attempt to find the separation of a star from an isochrone comes from Schaltenbrand (1974) who developed a simple method to estimate the nearest point of the zero-age main sequence to a given star in a two-colour diagram under the assumption of Gaussian errors. This approach was further formalised in a proper probabilistic framework by Luri, Torras \& Figueras (1992) as the so-called 'proximity parameter', similar to the deterministic 'near point' estimator by Flannery \& Johnson (1982). Here one computes the sum of minimum distances from a set of $N_{\star}$ stars to a given isochrone with given properties (age $t$, Helium abundance $Y$, metal content $Z$, etc) along with properties of the set of stars, which can also be applied to the theoretical isochrone, such as the distance modulus $\mu$ or the extinction. We represent this set as the vector of parameters $\vec{\vartheta}=(t, Y, Z, \mu, \cdots)$. One can form the statistic $\Psi^{2}$ which is calculated

\footnotetext{
${ }^{2}$ The spectra of the fainter members of the Pleiades came from E.C. Pickering and A. Cannon, and no mention is made of Rosenberg (1910)'s work nor the comprehensive survey by Hertzsprung (1911).
} 
as the sum of minimal (squared) distances:

$$
\Psi^{2}(\overrightarrow{\boldsymbol{\vartheta}})=\sum_{i=1}^{N_{\star}} \min _{j}\left(d_{i j}^{2}\right)
$$

where $d_{i j}$ is the geometrical distance between the observed star number $i$ and the theoretical point $j$ of the given isochrone:

$$
d_{i j}^{2}=\left(\frac{m_{i}-m_{j}}{\sigma\left(m_{i}\right)}\right)^{2}+\left(\frac{c_{i}-c_{j}}{\sigma\left(c_{i}\right)}\right)^{2}
$$

and $m$ and $c$ are magnitudes and colours respectively, or gravities and temperatures, or any two observables which can be predicted with the models, while $\sigma\left(m_{i}\right)$ and $\sigma\left(c_{i}\right)$ are the errors in these quantities for the given star labeled $i$. This distance can obviously be generalised to any $n$-dimensional space (see below). If (and this is a major assumption, see below) the model stars were uniformly distributed along the isochrone, then the probability that one observed star comes from that isochrone would be

$$
\mathcal{P}_{i}(\overrightarrow{\boldsymbol{\vartheta}})=\frac{1}{2 \pi \sigma\left(m_{i}\right) \sigma\left(c_{i}\right)} \sum_{j=1}^{N_{i s o}} \exp \left(-d_{i j}^{2} / 2\right),
$$

where $N_{\text {iso }}$ is the total number of points the isochrone has been divided into. The probability that an ensemble of $N_{\star}$ stars comes from an isochrone with the set of properties $\vec{\vartheta}$ is just the product of the individual probabilities

$$
\mathcal{P}_{\text {total }}(\overrightarrow{\boldsymbol{\vartheta}})=\prod_{i=1}^{N_{\star}} \mathcal{P}_{i}(\overrightarrow{\boldsymbol{\vartheta}})
$$

One can then use the standard maximum likelihood technique to find the set of parameters $\vec{\vartheta}$ which maximises this probability. The maximum likelihood statistic (MLS) would for instance be

$$
\operatorname{MLS}(\overrightarrow{\boldsymbol{\vartheta}})=-\log \mathcal{P}_{\text {total }}(\overrightarrow{\boldsymbol{\vartheta}})=\sum_{i=1}^{N_{\star}} \log \mathcal{P}_{i}(\overrightarrow{\boldsymbol{\vartheta}})
$$

For, say, $m=3$ parameters (distance modulus, age, and metallicity) this would be an optimisation problem: find the point in this 3-dimensional space for which MLS reaches a maximum.

The procedure is best illustrated for the case of a single star $\left(N_{\star}=1\right)$, as shown in Fig. 1 where a star (and its errors, which determine the elliptical confidence region) is represented in a CMD along with two possible isochrones of very different properties but which lie at the same normalised distance $d_{1 m}$ of unity. Clearly many more isochrones can have the same normalised distance or even smaller ones, but they both show that one cannot decide which isochrones fits best, since both 
contribute the same amount to the $\Psi^{2}$ statistic or to the MLS one. The problem, thus formulated, is intrinsically degenerate, as there are multiple solutions: many different combinations of the basic parameters $\vec{\vartheta}$ can give the same maximum in the likelihood.

The reason for this degeneracy stems from the key assumption made in deriving the probability that the star was sampled from the isochrone (Eq. 3.3): it was assumed that the number of stars along the isochrone was the same. That is, we were only concerned about the geometrical shape of the isochrone, and not about the density of stars along it. This geometrical method, while useful in selecting shapes of isochrones which come close to the observed position, is highly degenerate (Fig. 1).

Figure 2 illustrates one aspect of this degeneracy: isochrones of widely different ages and metallicities have a very similar geometrical shape. It is therefore hardly surprising that geometrical methods which rely on the proximity of a star to these isochrones yield huge degeneracies. Adding the uncertainties in distance modulus and dust extinction makes these methods unsuitable for any quantitative analysis.

While extensions of this geometrical method are discussed in their contexts later on, it is worth understanding in more detail the underlying reason for which the distribution of stars is not uniform along an isochrone.

Let us consider a curvilinear coordinate $s$ along an isochrone of given parameters. Stellar evolution theory predicts that for a given abundance the number of stars on the isochrone depends only on the age $t$ of that isochrone and on the mass $m$ of the star at that precise locus, so that $s=s(m, t)$ only. An offset in age $d t$ is reflected only through changes in mass $m$ and position $s$ along the isochrone of age $t$ since

$$
d t(m, s)=\left.\frac{\partial t}{\partial m}\right|_{s} d m+\left.\frac{\partial t}{\partial s}\right|_{m} d s \quad .
$$

For a star in the isochrone the offset is, by definition, $d t=0$ hence

$$
\left.\frac{\partial m}{\partial s}\right|_{t}=-\left.\frac{\partial m}{\partial t}\right|_{s} \times\left(\left.\frac{\partial s}{\partial t}\right|_{m}\right)^{-1} .
$$

The first term is always finite. The second term is the evolutionary speed, the rate of change for a given mass $m$ of its coordinate along the isochrone when the age changes by some small amount. One can think of $s$ as representing some evolutionary phase, and so this term will be large when the phase is short-lived : a small variation in age yields a very large change in position along the isochrone. Alternatively, for a given age, and since the first term is always finite, a wide variation in position implies a narrow range in mass. This is the case of the red giant branch or the white dwarf cooling sequence, for instance. On the other hand, slowly evolving phases such as the main sequence have small evolutionary speeds and wide ranges in mass for a given interval along an isochrone. Clearly, the most important phases to discriminate between alternative ages and metallicities will be the post main-sequence ones, where the range of mass is small (and hence 


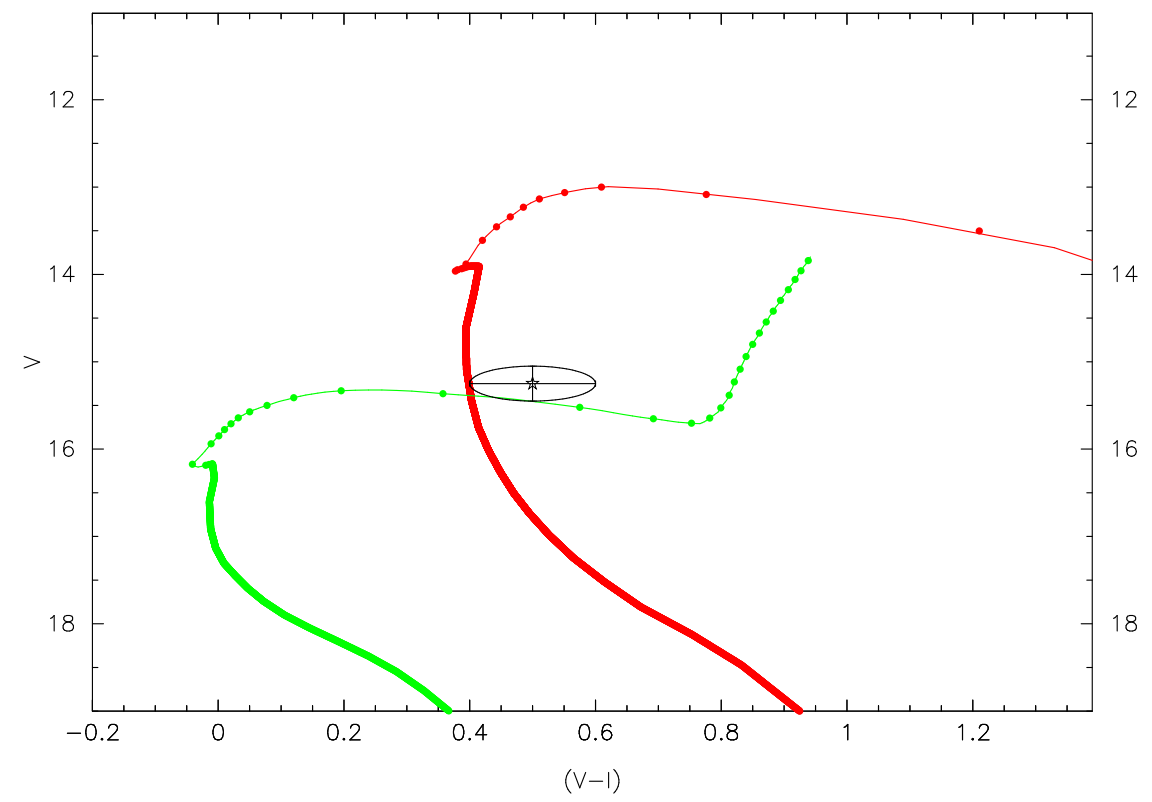

Fig. 1. The observation of a single star in a given CMD. Which of the two isochrones is more likely to be the correct one? Only two isochrones are plotted, for illustrative purposes, with different properties (age, distance, metallicity, etc), and the points along each isochrone are such that the diffence in stellar mass is the same (namely $\Delta m=$ $\left.0.01 M_{\odot}\right)$. In their main sequence the density of stars is very large (slow evolution) while after the turn-off, the much faster evolutionary speed makes the stars to become more widely separated for this fixed $\Delta m$. Many different isochrones can go through the same observed point (star with error bars at $1 \sigma$ and with the corresponding probability level given by the elliptical curve), and hence there are, in principle, many possible solutions: there is a huge degeneracy. For clarity, in this Figure only two isochrones are marked whose Near Point lie at a normalised distance of $1 \sigma$ from the observed position. The geometrical term of their likelihoods is identical (same distance in probability or $\sigma$ units), yet the lower (green) isochrone has an evolutionary term much smaller than the one from the upper (red) isochrone: the density of points from the lower (green) isochrone is much smaller when arriving close to the observed star. Hence, it is much less probable that the observed star is drawn from it. The purely geometrical degeneracy can be lifted using the prior information provided by stellar evolution in terms of the evolutionary speed of each track. In addition, another prior comes from the observed stellar mass function, which also favours smaller stellar masses. 

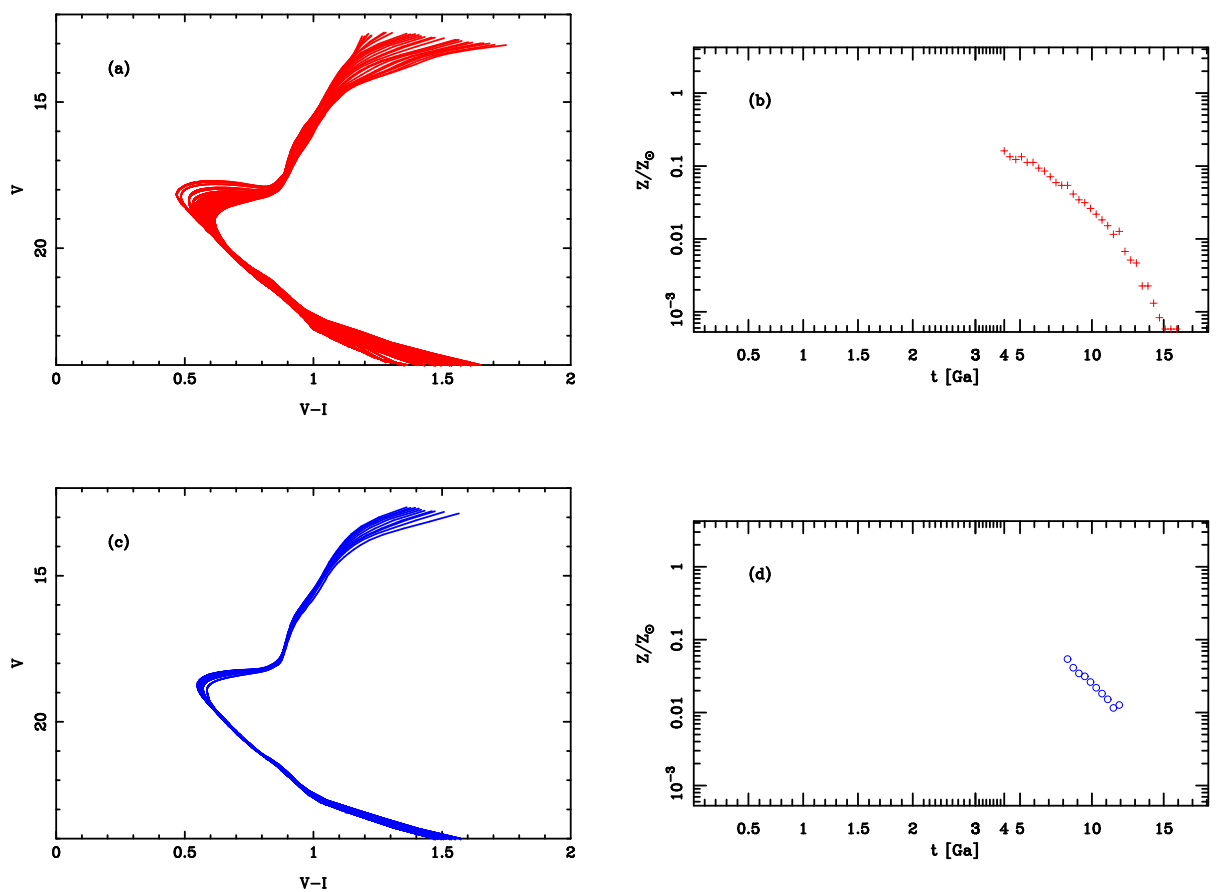

Fig. 2. An illustration of the geometrical degeneracy between age and metallicity. In panel (a) a series of isochrones of roughly similar shapes is indicated, whose ages and metallicities are given in panel (b). The well-defined locus in the $Z$-age plane means that many CMD inversions with sparse populations, differential reddening or binaries will tend to produce age-metallicity "relations" which only reflect this geometrical degeneracy. This is further illustrated in panel $(c)$ where the geometrical shapes are even closer. Yet, as panel $(d)$ shows, any isochrone changing its metallicity by nearly an order of magnitude can produce another isochrone of the very same geometrical shape provided the age changes by about a factor of two. Physically, there is no such degeneracy, as a change of (atmospheric) metallicity reflects a change in the (core) abundance, and hence a different evolutionary speed, as reflected by the density of stars along the isochrone.

insensitive to the details of the stellar mass function), and at the same time where evolutionary speeds are large. If we consider the mid- to lower main sequence, at fixed metallicity, isochrones of all ages trace the same locus, with only marginal changes in the density distribution of points amongst them. In this sense, one of the parameters, the age $t$, is to a large extent absent from the main sequence, while phases beyond it are always substantially a function of (at least) both age and metallicity. 
The density of stars along an isochrone is therefore

$$
\frac{d N}{d s}=-\left(\frac{d N}{d m}\right) \times\left(\left.\frac{\partial m}{\partial t}\right|_{s}\right) \times\left(\left.\frac{\partial t}{\partial s}\right|_{m}\right) \quad .
$$

The first term is related to the initial stellar mass function (IMF), and, as discussed above, the second term is finite while the third is a strong function of the evolutionary speed. If the mass after the turn-off is assumed to be roughly constant, this implies that the ratio in the number of stars in two different evolutionary stages after the turn-off will only depend on the ratio of their evolutionary time scales. In the context of stellar population synthesis, this is known as the fuel consumption theorem (Renzini \& Buzzoni, 1983).

The way to infer the properties of star, given some observables, and our knowledge of stellar evolution, is obviously the Bayesian method, where both the errors in the observables and our prior information (stellar evolution) can be handled properly, even in the case of one single star. Good reviews of Bayesian inference in physics are provided by Cousin (1995), Dose (2003) and Trotta (2008), along with the monographs by Gregory (2005) and Hobson et al. (2010). The Bayesian method allows us to answer the question we are interested in: What is the probability of the occurence of the estimated parameters $\vec{\vartheta}$ (mass, distance, age, etc) given the observed dataset $\overrightarrow{\mathbf{D}}=(V, V-I, B-R, \cdots)$ and our prior information provided by stellar evolution? The prior information can be formulated as the probability distribution function expected for the parameters, $\pi(\vec{\vartheta})$, on the basis of stellar evolution or other prior knowledge (say, a measure of $Z$ ), and is normalised to one, $\int \pi(\overrightarrow{\boldsymbol{\vartheta}}) d \overrightarrow{\boldsymbol{\vartheta}}=1$. The probability of observing the dataset $\overrightarrow{\mathbf{D}}$, given some parameters $\overrightarrow{\boldsymbol{\vartheta}}$ is the likelihood $\mathcal{L}(\overrightarrow{\mathbf{D}} \mid \overrightarrow{\boldsymbol{\vartheta}})$. An important quantity is the evidence (sometimes also termed as marginal likelihood) which is just $E(\overrightarrow{\mathbf{D}})=\int \pi(\overrightarrow{\boldsymbol{\vartheta}}) \mathcal{L}(\overrightarrow{\mathbf{D}} \mid \overrightarrow{\boldsymbol{\vartheta}}) d \overrightarrow{\boldsymbol{\vartheta}}$. Bayes (1763)'s theorem ${ }^{3}$ then states that

$$
\mathcal{P}(\overrightarrow{\boldsymbol{\vartheta}} \mid \overrightarrow{\mathbf{D}})=\pi(\overrightarrow{\boldsymbol{\vartheta}}) \frac{\mathcal{L}(\overrightarrow{\mathbf{D}} \mid \overrightarrow{\boldsymbol{\vartheta}})}{E(\overrightarrow{\mathbf{D}})}
$$

In other terms, our prior information of the parameters is modified into our posterior probability distribution function by the ratio of the likelihood over the evidence. We will see that this formulation of the problem also allows us to discriminate among models through the model selection technique. In the case of an observed star to be associated with an isochrone with $n$ observables $\overrightarrow{\mathbf{D}}$ and $n^{\prime}$ parameters $\vec{\vartheta}$, we can define a simple geometrical likelihood as

$$
\mathcal{L}_{\text {geom }}(\overrightarrow{\mathbf{D}} \mid \overrightarrow{\boldsymbol{\vartheta}})=\frac{1}{(2 \pi)^{n / 2} \prod_{k=1}^{n} \sigma_{k}} \prod_{k=1}^{n} \exp \left(-D_{k}^{2} / 2\right)
$$

\footnotetext{
${ }^{3}$ Dale (1982) explores the issue of whether Laplace (1812) should rather been given credit to the actual use, proof and development of the theorem.
} 
where $D_{k}$ is again the normalised distance between the observed quantity $S_{k}$ and the one predicted by the model $M_{k}$, given an observed error $\sigma_{k}$ in that quantity

$$
D_{k}^{2}=\left(\frac{S_{k}-M_{k}}{\sigma_{k}}\right)^{2} .
$$

For example, we can take $n=3$ observables such as $\overrightarrow{\mathbf{D}}=\left(m_{V}^{o b s}, T_{\mathrm{eff}}^{o b s}, Z^{\text {obs }}\right)$, which depend on, say, $n^{\prime}=5$ theoretical parameters such as $\vec{\vartheta}=(m, t, d, Z, \alpha)$ and we would have

$$
\begin{gathered}
\mathcal{L}_{\text {geom }}\left(m_{V}^{o b s}, T_{\text {eff }}^{o b s}, Z^{o b s} \mid m, t, d, Z, \alpha\right)= \\
\frac{\exp \left(-\chi^{2} / 2\right)}{(2 \pi)^{3 / 2} \sigma\left(m_{V}^{o b s}\right) \sigma\left(T_{\text {eff }}^{o b s}\right) \sigma\left(Z^{o b s}\right)},
\end{gathered}
$$

with, under the assumption that they are uncorrelated,

$$
\begin{aligned}
\chi^{2} & =\left(\frac{m_{V}^{o b s}-m_{V}^{\text {theo }}(m, t, d, Z, \alpha)}{\sigma\left(m_{V}^{o b s}\right)}\right)^{2} \\
& +\left(\frac{T_{\mathrm{eff}}^{o b s}-T_{\mathrm{eff}}^{\text {theo }}(m, t, d, Z, \alpha)}{\sigma\left(T_{\mathrm{eff}}^{o b s}\right)}\right)^{2} \\
& +\left(\frac{Z^{o b s}-Z^{\text {theo }}(m, t, d, Z, \alpha)}{\sigma\left(Z^{\text {obs }}\right)}\right)^{2}
\end{aligned}
$$

If quantities are correlated, one has to form the pairs

$$
\mathcal{L}_{\text {corr }}(A, B)=\frac{\exp \left(-\left[D_{A}^{2}+D_{B}^{2}-2 \rho D_{A} D_{B}\right] /\left[2\left(1-\rho^{2}\right)\right]\right)}{2 \pi \sigma(A) \sigma(B) \sqrt{1-\rho^{2}}},
$$

where $\rho$ is the correlation coefficient between the two quantities $A$ and $B$, or, in terms of their covariance, $\operatorname{cov}(A, B)=\rho \sigma(A) \sigma(B)$.

We can illustrate this case with an example where we assume to have a prior knowledge of the distance of the star and its bolometric correction so that we can for instance use the bolometric luminosity rather than its apparent magnitude in some photometric band.

Fig. 3 shows as a test case five stars with the same effective temperature, but different luminosities so as to sample different evolutionary régimes (top panel). There is no prior information on age or metallicity nor mass. If we are only interested in, say, the ages of these stars, we can consider the mass, the metallicity, the mixing length parameter, etc, as nuisance parameters with flat probability distributions (say, between 0.2 and $200 M_{\odot}, 0.0001$ and 0.2 , and 0.5 to 2.0 , respectively) so that the probability distribution function (PDF) of the age is given by

$$
\mathcal{P}(t)=\iiint d m d Z d \alpha \mathcal{L}_{\text {geom }}(t, Z, m, \alpha)
$$




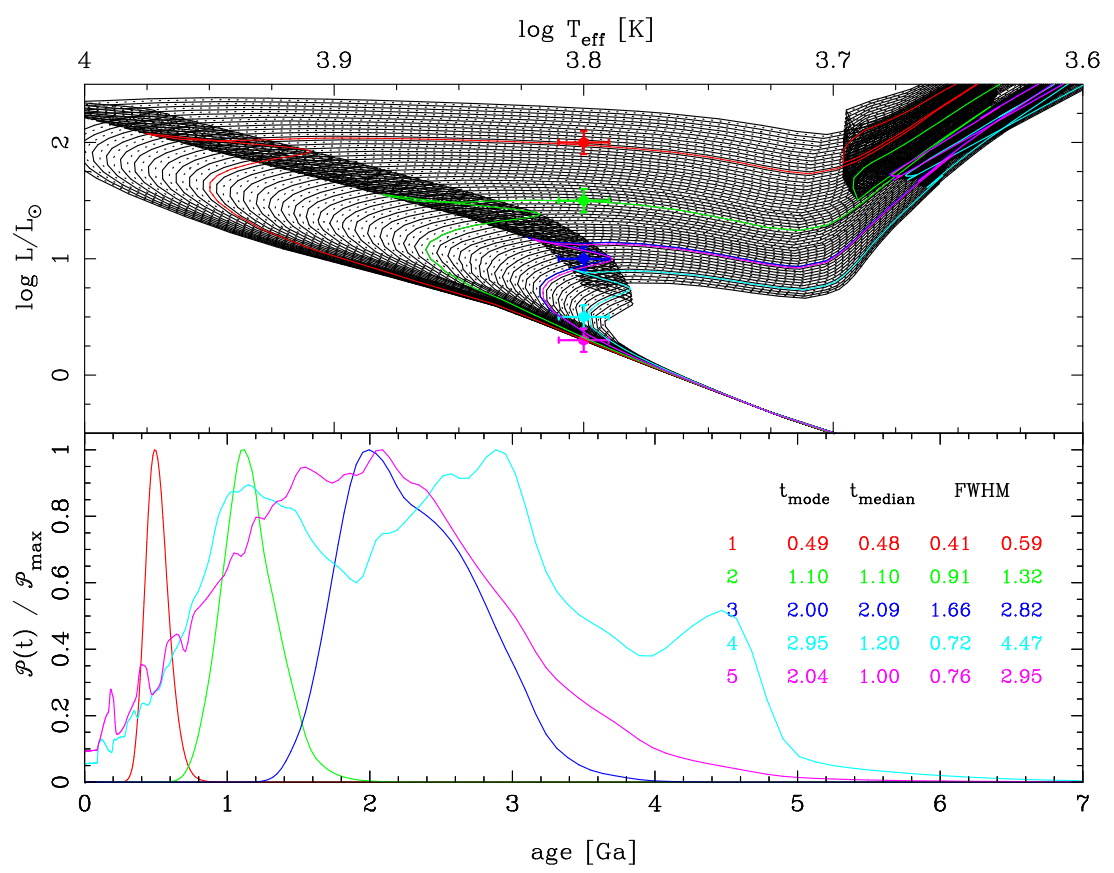

Fig. 3. A test case with 5 stars at fixed effective temperature $\left(\log T_{\text {eff }}=3.8, \sigma\left(\log T_{\text {eff }}\right)=\right.$ 0.01) and five different bolometric luminosities sampling the HRD in regions where: (i-ii) evolution is fast (top two stars, colour-coded red and green); (iii) near the TAMS, where multiple isochrones cross (blue star $\left(\log L / L_{\odot}=1.0\right)$; (iv) a slightly evolved star away from the MS (pale blue); and ( $v$ ) a star very close to the ZAMS traced by very young stars. The lower planel shows the posterior probability distribution function for the age of each star, assuming flat priors for the other parameters. In this figure, the likelihood is computed using the geometrical term only, and while the upper two stars appear to have well-defined ages (a FWHM range from 0.41 to $0.59 \mathrm{Ga}$ for the first star, and from 0.91 to $1.32 \mathrm{Ga}$ for the second one), the star located between the red and the blue loops has a FWHM range of $1.5 \mathrm{Ga}$, and it gets worse for the two lower stars. This is just due to the large density of isochrones in these areas, and the geometrical term of the likelihood cannot disentangle, per se, which set is more appropriate, since no prior information on the evolutionary speed is used.

Likewise, if we are interested in estimating the mass of that star, we would marginalise over the other (nuisance) parameters to get

$$
\mathcal{P}(m)=\iiint d t d Z d \alpha \mathcal{L}_{\text {geom }}(t, Z, m, \alpha)
$$

We illustrate the technique here for the ages of the stars, given their importance in 
the context of both CMDs and stellar evolution (e.g., Lebreton, 2000; Soderblom, 2010), and because they have been widely used, even though they are not physically justified (e.g Lachaume et al., 1999; Reddy et al., 2003). The resulting age PDFs are given in the lower panel of Fig. 3 and show the widely different distributions depending on the location of the test stars in the diagram (all share the same errors in bolometric luminosity and effective temperature, for the sake of the argument).

More importantly, however, this formulation of a geometrical likelihood (Eq. 3.10) does not include any information on the evolutionary speed: we have only used the geometrical shape of the isochrone, the only quantity that matters when computing the distance from the observed star to a point on the isochrone (Eq. 3.11). To incorporate the physics of stellar evolution we need to account for the density of stars along the isochrone (Eq. 3.8) so that we have a proper physical likelihood. To do this, we need to integrate along all possible masses in the isochrone, but noting that (unlike the geometrical case) not all masses are equally probable. Let the density of stars of mass $m$ along an isochrone be $\rho(m)$, then

$$
\mathcal{L}_{\text {phys }}(\overrightarrow{\mathbf{D}} \mid \overrightarrow{\boldsymbol{\vartheta}})=K^{-1} \int_{m_{\text {lim }}}^{m_{\text {top }}} d m \frac{\rho(m)}{(2 \pi)^{n / 2} \prod_{k}^{n} \sigma_{k}} \prod_{k}^{n} \exp \left(-D_{k}^{2} / 2\right)
$$

where, for a proper normalisation, we require

$$
K=\int_{m_{\text {lim }}}^{m_{\text {top }}} d m \rho(m)
$$

and $m_{\text {lim }}$ and $m_{\text {top }}$ are the lower and upper mass limits of the isochrone considered. It is useful to write this in terms of the curvilinear coordinate $s$ along the isochrone, since the mapping of the mass $m$ to a position in the CMD is highly non-linear (cf.Eq. 3.8): the red giant branch will be poorly sampled if the mass interval is too large. We can thus re-write Eq. 3.17 as

$$
\mathcal{L}_{\text {phys }}(\overrightarrow{\mathbf{D}} \mid \overrightarrow{\boldsymbol{\vartheta}})=\int_{s=0}^{s=1} \underbrace{d s}_{\text {curvilinear }} \underbrace{\frac{d N(m)}{d m}}_{\phi(m)} \underbrace{\frac{d m}{d s}}_{\text {speed }} \underbrace{\frac{\prod_{k}^{n} \exp \left(-D_{k}^{2} / 2\right)}{(2 \pi)^{n / 2} \prod_{k}^{n} \sigma_{k}}}_{\text {geometry }},
$$

where the lower and upper limits of the curvilinear coordinate $s$ have been set to 0 and 1 respectively, and we can identify, besides the geometrical term, the initial mass function $\phi(m)$ and the evolutionary speed $d m / d s$ along the isochrone. These two functions encapsulate the prior information provided by stellar evolution, in a way that the geometrical approximation cannot possibly handle. In reference to previous works (Hernandez et al., 1999; Jørgensen \& Lindegren, 2005), we will refer this method as the BayesGM method ${ }^{4}$.

\footnotetext{
${ }^{4}$ In Hernandez et al. (1999) the physical likelihood was referred to as the G matrix.
} 
The posterior PDF then becomes

$$
\mathcal{P}_{\text {post }}(\overrightarrow{\boldsymbol{\vartheta}} \mid \overrightarrow{\mathbf{D}})=\prod_{k=1}^{n} \pi(\overrightarrow{\boldsymbol{\vartheta}})_{k} \prod_{i=1}^{N_{\star}} \mathcal{L}_{\text {phys }}(\overrightarrow{\mathbf{D}} \mid \overrightarrow{\boldsymbol{\vartheta}})_{i}
$$

where $\pi(\vec{\vartheta})_{k}$ is the prior probability distribution function for the parameter indexed $k$ of the parameter vector $\overrightarrow{\boldsymbol{\vartheta}}$. The key point of this formulation is that the prior on mass, the IMF $\phi(m)$, must be included in the likelihood, for a proper physical weighting. As the dataset is fixed, we do not need to compute the evidence which only acts, in this context, as a normalisation constant.

For example, consider a star on the red giant branch: for a long interval along the nearly vertical isochrone, the mass hardly changes, and hence the effective weight of the IMF in the integrand will be very small and the geometrical distance will, in proportion, be more important. In the main sequence the reverse is true. The effects of including explicitely in the likelihood this prior information are dramatic, and shown in Fig. 4. There is little difference for the two brightest stars, as isochrones run almost parallel to the effective temperature axis, and hence map the plane in a well-behaved way: the only difference with the geometrical method is that our prior information on both evolutionary speed and the IMF will favour tracks with smaller masses (they evolve more slowly and are more abundant, two factors that cannot be handled by the geometrical method). The middle star (colour-coded in blue) is more interesting as it lies in the area where multiple isochrones cross each other, hence providing a huge geometrical degeneracy as reflected by the age range from 1.66 to $2.82 \mathrm{Ga}$ (Fig. 3). In this particular case, large ages appear to be penalised, and the new FWHM range is restricted to 1.66 to $2.29 \mathrm{Ga}$ (Fig. 4). The next star illustrates this effect even more clearly, with a reduction of the interval $0.72-4.47 \mathrm{Ga}$ to $1.48-3.55 \mathrm{Ga}$, a contraction of 1.75 Ga. The faintest star, which appeared deceptively well behaved when using the geometrical method, now reveals that much younger ages are twice more probable, as well as older ones. The full prior information on the way stars evolve is properly used.

In Figs. 3 and 4 the full posterior age distributions are given. Rather than using the full PDF of the parameters, it is customary to encapsulate the information in quantities such as the (posterior) modes (the values of the parameters where the posterior PDF reaches a maximum), the means or expectation values, or even the medians. The frequentist confidence intervals become, within the Bayesian framework, the credible regions (CRs) such that they are the (closed but not necessarily connected) volumes which contain a fraction $\alpha$ of the total volume under the posterior:

$$
\int_{C R(\alpha)} d \overrightarrow{\boldsymbol{\vartheta}} \mathcal{P}_{\text {post }}(\overrightarrow{\boldsymbol{\vartheta}} \mid \overrightarrow{\mathbf{D}})=\alpha
$$

There are many different possible CRs. The central credible interval (CCI) is defined such that the intervals $\left(-\infty, \theta_{\text {low }}\right)$ and $\left(\theta_{\text {high }},+\infty\right)$ each contains $(1-\alpha) / 2$ 


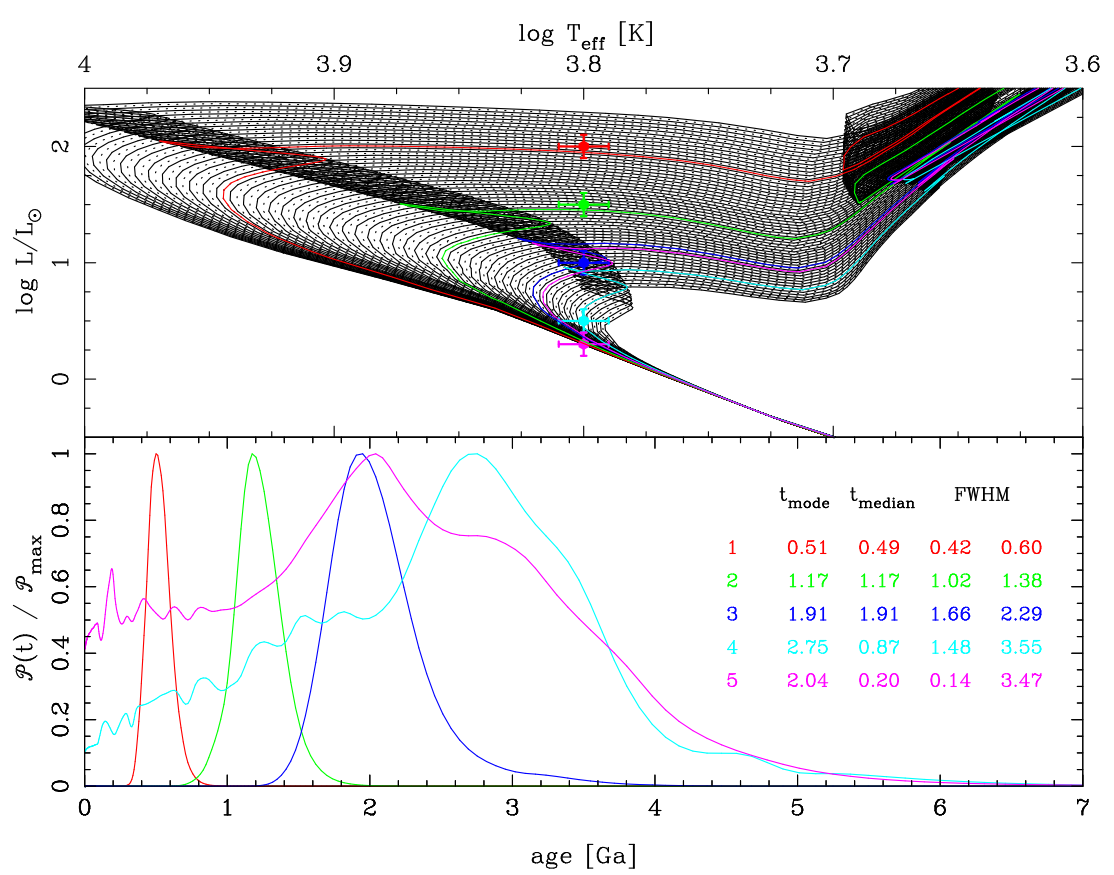

Fig. 4. The same set of stars as in Fig. 3. This time the full likelihood function (that is, with the evolutionary terms) and the prior on the mass function is used. There is a tendency to increase the mode and the median age for the two top stars, although still within the same FHWM range. This slight increase is due to the fact that the probability of observing a less massive star is larger, and hence the ages increase. This effect helps reducing the range for star \# 3 by almost $0.5 \mathrm{Ga}$, and produces well-defined peaks for the two lower stars, reducing the range of the posterior PDF. The effect is even stronger when a wide range of metallicities is considered: the posterior PDF peaks at the proper ages and metallicities, hereby lifting the purely geometrical degeneracy illustrated in Fig. 2.

of the posterior volume, and always contains the median. The minimum credible interval (MCI) is built in such as way tha the posterior PDF is always larger inside the MCI than outside. It contains the mode, obviously, but may not be connected. In Fig. 4 the FWHM (that is, the values for which the PDF reaches half its maximum value) are indicated, but note that they do not correspond to any fixed probability measure. The figure also illustrates the poor performance of the median when the PDF is wide. Similarly, and contrary to the claims by Burnett \& Binney (2010), the mean can be highly biased (although easy to compute). We hence prefer to use the mode of the distribution, as a robust point estimate of the quantity of interest. 


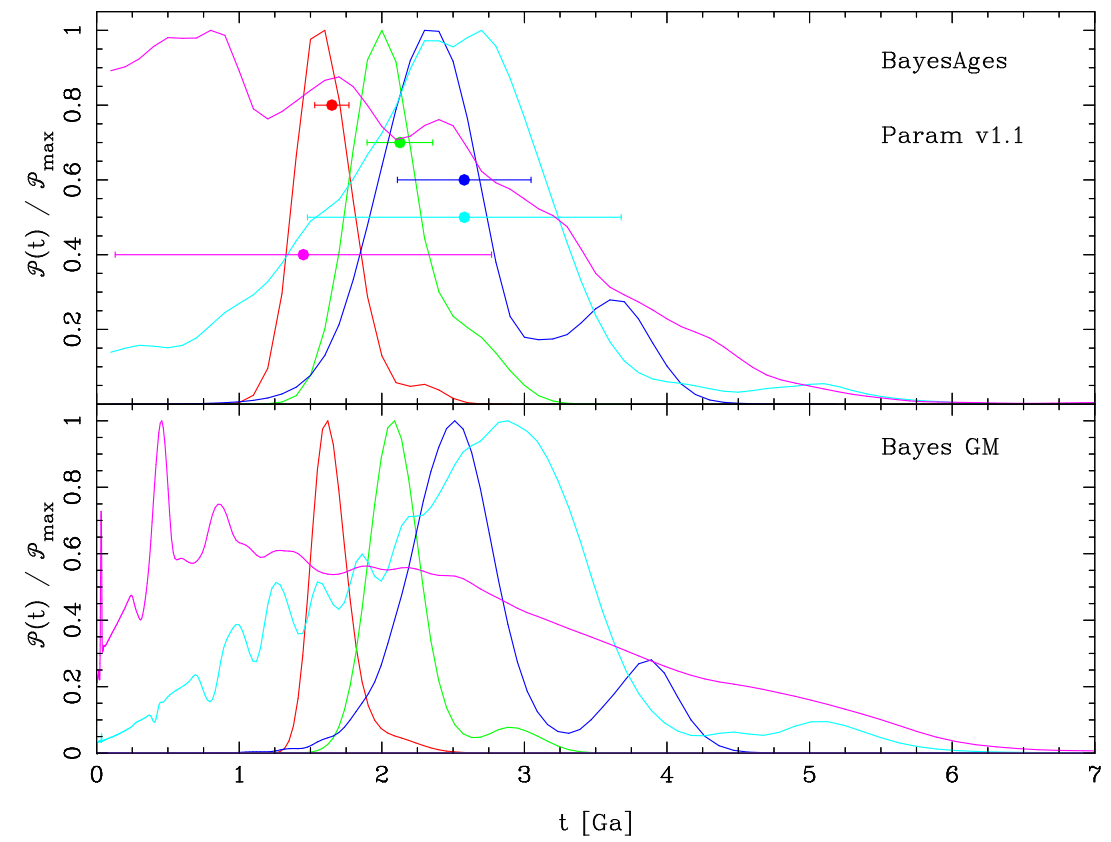

Fig. 5. Comparing the posterior PDF in ages for the 5 stars indicated in the top panels of Figs. 3 and 4, with uncertainties of $\sigma\left(\log T_{\text {eff }}\right)=0.01$ and $\sigma\left(M_{V}\right)=0.1$, this time at $M_{V}=2.0,2.5,3.0,3.5$ and 4.0. BayesAges (Pont \& Eyer, 2004) tends to produce slightly wider PDFs than our BayesGM technique, while PARAM v1.1 (Da Silva et al., 2006) yields point estimates slightly older ( $1 \sigma$ bars have been offset vertically for clarity). The welldefined peak at $t \approx 0.45$ Ga predicted by BayesGM, and which is not present in BayesAges is robust: decreasing the uncertainties in the two observables produces a mode at this age.

Pont \& Eyer (2004) have also developed a Bayesian technique to explore the distribution of ages for the particular case of $\mathrm{G}$ dwarf stars to revise the agemetallicity relation in the Galactic disc. They use a geometrical likelihood in the 3-dimensional parameter space of effective temperature, absolute magnitude and metallicity, and include stellar evolution is terms of priors (for instance, taking the variation of the luminosity as a function of age at fixed temperature). Fig. 5 compares the results of their method, BayesAge, with BayesGM for five test stars. While, as expected, the results are very similar for the brightest stars, for the fainter ones the bias they observe towards younger ages is not present in BayesGM. For the faintest test star, the peak predicted by BayesGM is confirmed when decreasing the error bars in the observables, confirming the robustness of the method. 
Da Silva et al. (2006) use a physical likelihood but take the IMF as describing the number of stars along a given isochrone, which is not correct (except on the ZAMS, see Eq. 3.8). Nevertheless, the estimates obtained agree with BayesGM while they seem to be systematically larger than BayesAges, as indicated in Fig. 5. The code PARAM v1.1 is available at stev.oapd.inaf.it/cgi-bin/param. Remarkably, Valenti \& Fischer (2005) did use what we could call a 'empirical' bayesian technique to weigh geometrically-estimated ages through a variety of 'probabilities' in a purely empirical way. In contrast, Takeda et al. (2007) used a proper Bayesian formalism which includes the possible variation of $\Delta Y / \Delta Z$ in stellar tracks, and limit the integration of the posterior PDF to the hyper-box in the space of parameters.

Breddels et al. (2010) apply a maximum likelihood technique again using a purely geometrical criterion, while Burnett \& Binney (2010) develop a Bayesian method to infer the first two moments of the posterior PDFs, again with a purely geometrical likelihood but properly weighted by priors. In contrast, Casagrande et al. (2011), building upon their previous work (Casagrande et al., 2010), include explicitely priors which attempt to correct for biases known to exist in their sample and a proper physical likelihood. Bailer-Jones (2011) also uses a Bayesian framework to estimate extinction and stellar parameters from measures of parallaxes and multi-band photometry. The prior information from stellar evolution is included explicitely by a distribution obtained with 200,000 stars of solar metallicity sampled from a Salpeter IMF and a flat star formation history. The (smoothed) density (obtained via a Gaussian kernel) is used as a prior in absolute magnitude and temperature.

Isochrone ages have now the statistical framework to be inferred properly, and which can be used to calibrate gyrochronological ages (e.g., Chanamé \& Ramírez, 2012), and be compared with the independent measures obtained through asteroseismology (e.g., Stello et al., 2009). The field has evolved dramatically since the first attempts (e.g., Perrin et al., 1977) with purely empirical fits to massive surveys with proper statistical methods (e.g., Nordström et al., 2004). The advent of large-scale surveys with measures of fundamental parameters, such as RAVE (e.g., Zwitter et al., 2010) and GAIA will show the usefulness of these Bayesian techniques.

\subsection{Caveats and (some) systematics}

There are two important caveats worth keeping in mind, besides the ones noted in $\S 1$ (photometric corrections, bolometric corrections and standard values) as there are two underlying assumptions that have been made: first that the light received in the detector actually corresponds to the star, and second that the position of the star in the CMDs is only determined by its mass, for a given age and metallicity. The first assumption could be wrong if the detected star is, in fact, an unresolved binary or multiple system. In this case, we are detecting the combined light from the components of the system, and both the magnitude and the colour are shifted by an amount which depends on the mass ratio(s). In the theoretical diagram, 
the luminosity and effective temperature of the combined system is related to the individual properties as

$$
\begin{aligned}
L_{A+B+C+\cdots} & =L_{A}+L_{B}+L_{C}+\cdots \\
T_{\mathrm{eff}(A+B+C+\cdots)}^{4} & =\frac{L_{A}+L_{B}+L_{C}+\cdots}{4 \pi \sigma\left(R_{A}^{2}+R_{B}^{2}+R_{C}^{2}+\cdots\right)},
\end{aligned}
$$

and not, as some authors have wrongly claimed (Siess et al., 1997), as the luminosityweighted mean of the effective temperatures. In the CMDs this effect gives rise to well known offsets from single star sequences (e.g., Haffner \& Heckmann, 1937; Maeder, 1974; Lastennet \& Valls-Gabaud, 1996; Hurley \& Tout, 1998) and unless one has good reasons to assume the star in consideration is truly single, the effect will produce a systematic bias in the inferred properties.

The second assumption covers several effects. The first one is just a consequence of Vogt (1926)'s theorem, which is not valid for instance in cooling white dwarfs, where the complicated details of the cooling sequence no longer depend only on the mass. In principle, the parameters controling the locus of the white dwarf in the cooling sequence could be included in the multivariate set $\overrightarrow{\boldsymbol{\vartheta}}$, and the same formalism can be applied (von Hippel, 2005; von Hippel et al., 2006; Jeffery et al., 2007; van Dyk et al., 2009; Jeffery et al., 2011). The same applies to pre-mainsequence stars, whose tracks depend to a large extent on their mass accretion history, which introduces a major complication, with, in principle, a functional degree of freedom which is difficult to constrain (Mayne \& Naylor, 2008; Naylor, 2009). Attempts are currently being made to use a Bayesian formalism to tackle this problem (Gennaro et al., 2012).

An equally serious case where the assumption is known to be wrong is provided by massive stars, whose fast rotations not only bring fusion products from the core to their surface during core hydrogen burning, and hence affect the abundances, but also their location in a CMD depends at least both on the inclination and on the equatorial velocity, none of which are measurable (only $v \sin i$ can be inferred from the line profiles). The effects of rotation can reach some 0.1 mag or more in both colour and magnitude (Maeder \& Peytremann, 1970; Collins \& Sonneborn, 1977). While statistical techniques (e.g., Collins \& Smith, 1985; Lastennet \& VallsGabaud, 1996) could be used incorporating further parameters into the $\overrightarrow{\boldsymbol{\vartheta}}$ vector, the availability of state-of-the-art models of (massive) rotating stars (e.g., Brott et al., 2011; Maeder \& Meynet, 2012) may provide another way of dealing with stars in the upper main sequence or beyond.

The third effet is the assumed enrichment $\Delta Y / \Delta Z$ which is built-in in the evolutionary tracks. Clearly different assumptions on both the Helium abundance $Y$ and the enrichment ratio have consequences on the stellar speed, and thus far only tests with two different sets have been carried out (Casagrande et al., 2011), but much more tests need to be done.

Last, but not least, is the thorny issue of the calibration of the mixing length convection theory (MLT): all tracks/isochrones are normalised to the putative solar case, and so stars with widely different masses and metallicities are still 
assumed to have the very same properties as the solar convective layers. While there is some mild evidence for a possible variation of the MLT parameter (which describes the mixing length in units of the local pressure scale) with mass in some binary systems (e.g., Lastennet et al., 2003; Yıldız et al., 2006; Yıldız, 2007) no isochrones have so far been computed for different scalings of the MLT parameter with mass and/or metallicity, and yet it is clear that stellar convection must depend on stellar parameters, as 3-dimensional simulations are indicating (Ludwig et al., 1999). Similarly the amount of overshooting is yet another degree of freedom which is rarely taken into account even if tracks are computed for a variety of possible values (e.g., VandenBerg et al., 2006).

All these provisos are worth keeping in mind before any interpretation of the resulting PDFs is carried out.

\section{Detached binary stars}

Binary stars can provide, in some circumstances, the only direct and reliable way to measure stellar masses and hence constitute a benchmark for stellar evolution. They can also provide accurate measures of many other quantities, and in some cases the full orbital and physical parameters. Astrometric (or interferometric) orbits combined with radial velocities, or detached eclipsing binaries which are also double-lined spectroscopic ones yield a wealth of precise measures reaching sometimes 1\% in masses and radii (see Torres, Andersen \& Giménez, 2010, for a comprehensive review). A catalogue of over 130 well-measured binary systems is mantained at www.astro.keele.ac.uk/jkt/debcat.

It is therefore quite natural to check how their own colour-magnitude diagrams can test stellar evolution, when the assumption is made that both components evolved independently of each other (i.e., there were no mass transfer episodes).

While there have been many attempts at using classical frequentist statistical methods (e.g., Lastennet \& Valls-Gabaud, 2002; Young et al., 2001; Malkov et. al, 2010 , and references therein), the Bayesian framework presented in the previous section allows one to infer more robustly the distribution functions of the parameters we are interested in. While a detailed analysis is beyond the scope of this chapter, we can easily check, as an example, the effects of different observables on the inferred ages of the components.

We use the BayesGM formalism to infer the posterior distribution function of age for each component of the well-studied binary system $\beta$ Aur (Torres, Andersen \& Giménez, 2010). Fig. 6 shows the resulting PDFs, with no prior assumption on their possible coevality. Their posterior modes coincide at an age of $0.41 \mathrm{Ga}$ when using effective temperatures combined with absolute magnitudes in the $V$ band. Fig. 7 shows the same posterior age distributions when another pair of observables is used: gravity and effective temperature. In this case the posterior PDFs are more concentrated, but their modes are significantly different from the ones using absolute magnitudes and temperatures. In spite of small bolometric corrections, using absolute luminosities rather than absolute magnitudes in $V$ yield wider PDFs (Fig. 8), with ages consistently larger than the ones inferred from the 
$\left(\log g, \log T_{\text {eff }}\right)$ pairs. Remarquably, in all cases the PDFs for each component overlap, arguing strongly for coevality (but note this was not assumed a priori). If we impose the condition of coevality, the likelihoods of each component are combined and multiplied by a Dirac distribution $\delta\left(t_{A}-t_{B}\right)$. The resulting PDFs are shown with solid lines in these three figures. As expected in the Bayesian framework, the more information included results in posterior distributions which are much narrower.

While there are no other independent constraints on age, one could use the isochrones to estimate the posterior PDFs on the masses, which are measured independently, or else use the measures as priors. This can very easily done in the definition of the physical likelihood.

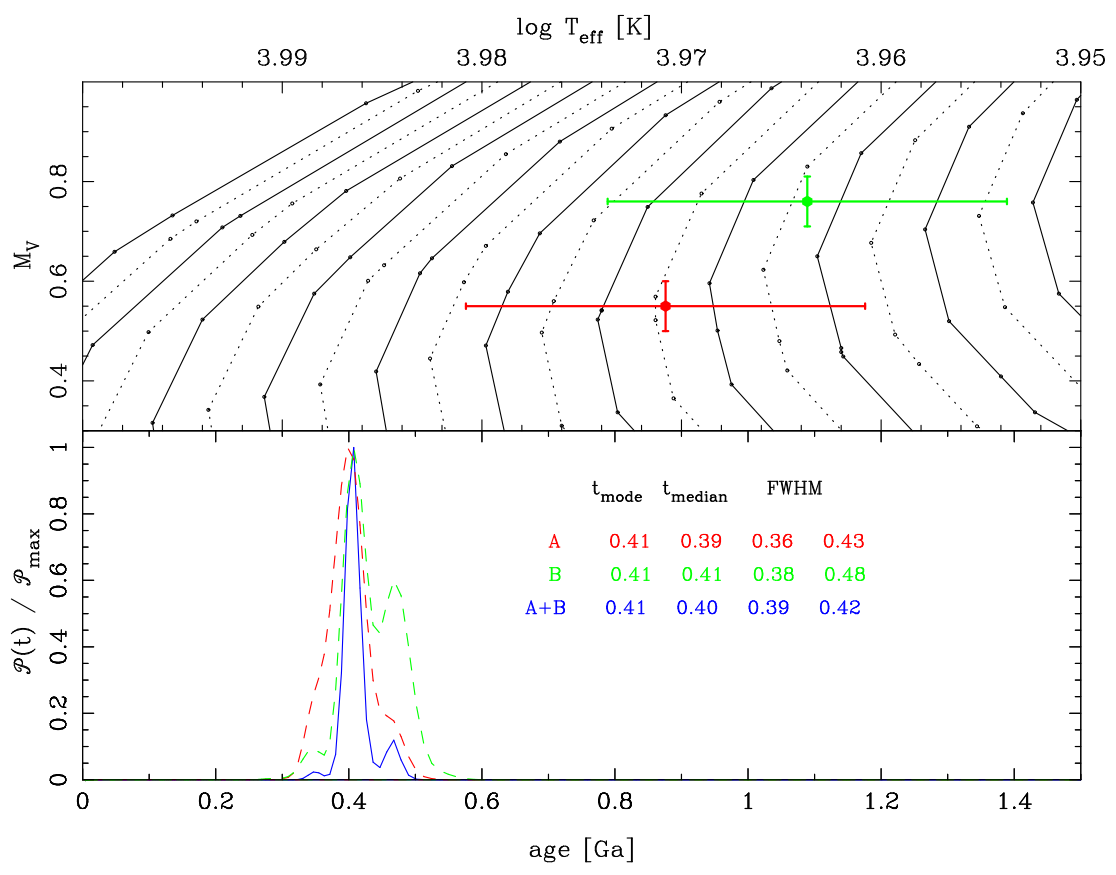

Fig. 6. Testing Bayesian ages in well-studied detached binary systems. Here we selected $\beta$ Aur to minimise the uncertainties in the bolometric corrections. The values for the parameters are given by Torres, Andersen \& Giménez (2010). The posterior PDF in age for component $\mathrm{A}$ is given by the red distribution, while the green curve gives the PDF for component B. In spite of a secondary peak in the latter, they have the same mode, and their medians are very similar. If we impose the constraint that they ought to have the same age, the prior is then $\pi(t)=\delta\left(t_{A}-t_{B}\right)$ resulting in a joint PDF given by the blue curve, with a much reduced FWHM range. 


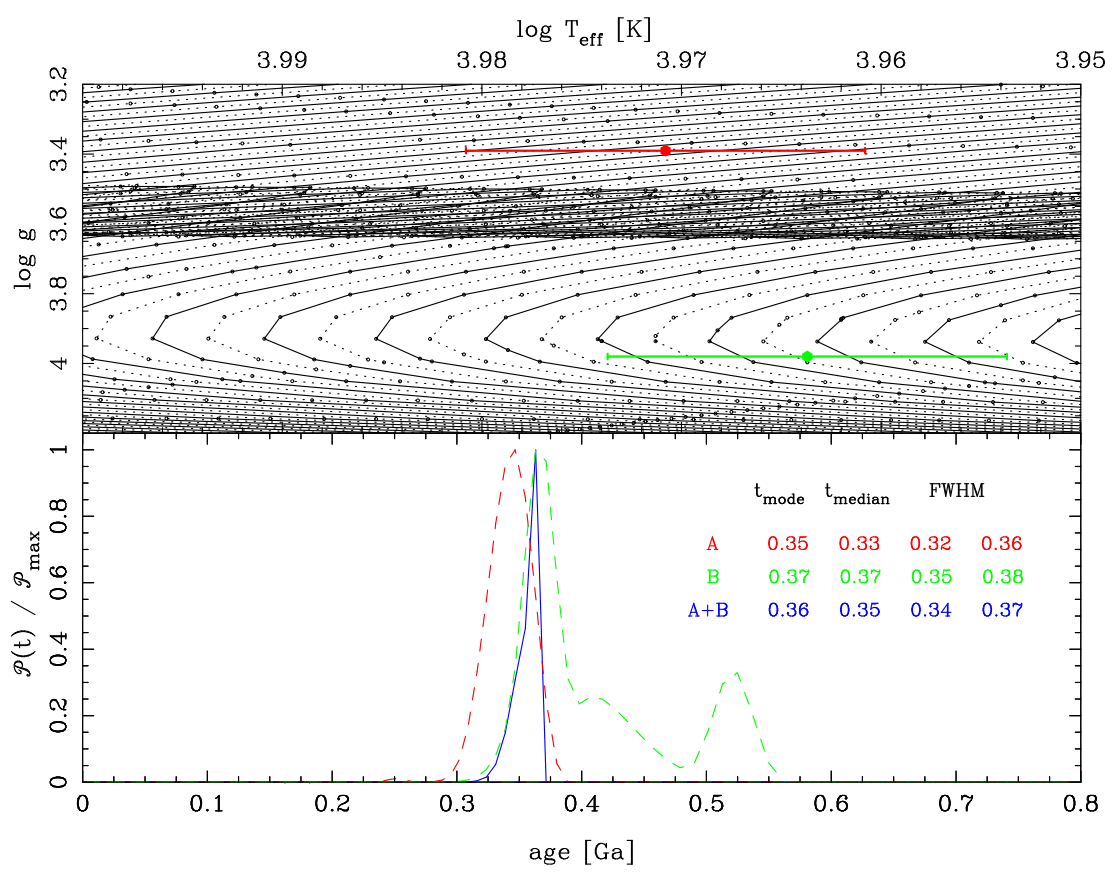

Fig. 7. The double-lined eclipsing binary $\beta$ Aur (as in Fig. 6) this time using effective temperatures and gravities, whose measures are assumed to be uncorrelated. The error bars $\sigma(\log g) \sim 0.005$ are too small to be seen at the scale of this diagram. While the posterior modes are different, the ranges are very similar. The joint distribution assuming coevality yields a modal age which is significantly younger than the one inferred using absolute magnitudes and effective temperatures (Fig. 6).

One can also combine prior information when binaries are known to be members of a cluster: as their share the same distance, one can impose this condition in the likelihood to get better constraints on the other parameters (e.g., Lastennet et al., 1999), even though in the case of very nearby clusters the depth or extent along the radial direction may be an issue.

Systematics are likely to become the major source of uncertainties in these analyses. Limb darkening 'laws' appropriate for the stars in consideration, the amount of 'third light', the sources of noise, etc (Southworth, 2011). Yet, the advent of both massive variability surveys and space missions is providing light curves of such quality (Bruntt \& Southworth, 2008) that empirical methods can no longer be reasonably used. 


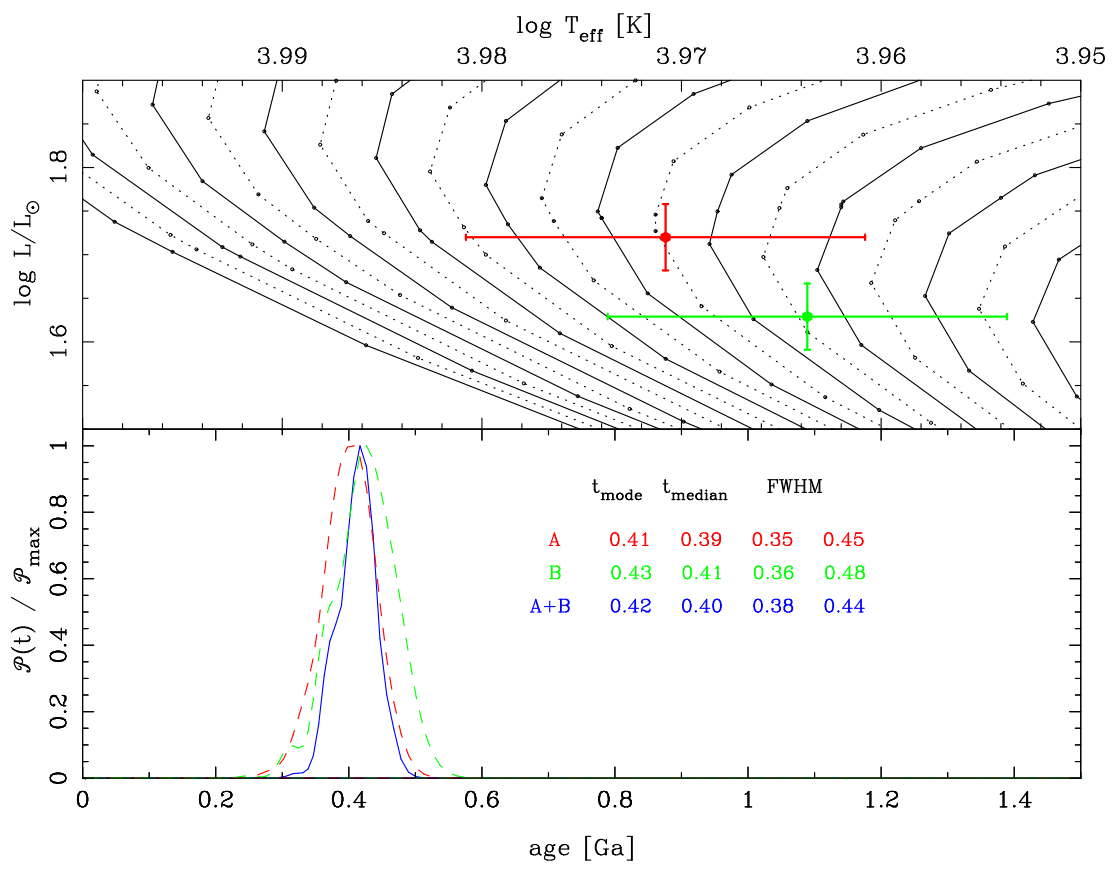

Fig. 8. Posterior PDF for the age of $\beta$ Aur, using effective temperatures and absolute luminosities, assumed to be uncorrelated. As the bolometric corrections at these temperatures are small, it is not surprising to get results similar to those using absolute magnitudes (Fig. 6), although the distributions appear to be wider.

\section{Coeval stellar populations}

The quantitative comparison of synthetic CMDs with observations relied initially on either post-MS phases, or the ratio of giants to main-sequence stars, the tips of various loops, etc (see, e.g., Meyer-Hofmeister, 1969; Robertson, 1974; Becker \& Mathews, 1983, for some early attempts). The advent of high-quality CCD photometry and the increase in computing power made it possible to apply proper statistical tools to the problem of inverting the observed CMDs to infer the underlying physical properties. Patenaude (1978) is an example in the attempt at setting up empirical isochrones for open clusters, for an easier comparison with theoretical ones. In fact, observers have been fond of establishing the so-called 'semi-empirical methods' whereby the separation between characteristic points in the CMD are calibrated with theoretical models. Hence we have for instance the vertical separation between the turn-off (TO) and the horizontal branch, or the horizontal separation between the TO and some point in the sub-giant branch. 
These distances are in fact ill-defined, as photometric errors, binaries, and the intrinsic sampling contribute to a dispersion which is difficult to quantity. Detailed attempts at calibrating these 'empirical' distances (e.g., Meissner \& Weiss, 2006) must be superseded by a proper modern statistical framework.

While producing synthetic CMDs used to be costly, there are many tools available based on different set of evolutionary tracks to create isochrones, luminosity functions, integrated magnitudes (Table 1).

Table 1. Some popular codes for generating isochrones and CMDs.

\begin{tabular}{ll}
\hline \hline CMD v2.6 & stev.oapd.inaf.it/cgi-bin/cmd \\
Victoria-Regina & \\
www2.cadc-ccda.hia-iha.nrc-cnrc.gc.ca/community/VictoriaReginaModels \\
Darmouth & stellar.dartmouth.edu/models \\
IAC-STAR & iac-star.iac.es/cmd/index.htm \\
\hline
\end{tabular}

Naylor \& Jeffries (2006) proposed a maximum likelihood method whereby a simulated underlying distribution function with some a priori information is made, and then maximise a geometric likelihood to assess a goodness of fit. The advantage of this approach is that it allows them to include populations of (unresolved) binaries, where both the fraction of binaries and the mass ratio distribution can be accounted for. Their code is available at the website www.astro.ex.ac.uk/people/timn/tau-squared.

The cross entropy technique has also been used in solving the optimisation problem (Monteiro et al., 2010), although in this case the authors used a geometrical likelihood, weighted through Monte Carlo realisations, which, in effect, reduce to the Bayesian formulation, although in a rather convoluted way.

We can use BayesGM to infer, for example, the age of a coeval stellar population just as we did for a binary system. We form the combined likelihood of the $N_{\star}$ stars with $n$ observables, say, $n=2$ with $(\mathrm{V}, \mathrm{B}-\mathrm{V})$, as

$$
\mathcal{L}_{\text {combined }}(V, B-V \mid \overrightarrow{\boldsymbol{\vartheta}})=\prod_{j=1}^{N_{\star}} \mathcal{L}_{\text {phys }}\left(V_{j},(B-V)_{j} \mid \overrightarrow{\boldsymbol{\vartheta}}\right)
$$

where $\mathcal{L}_{\text {phys }}(\overrightarrow{\mathbf{D}} \mid \overrightarrow{\boldsymbol{\vartheta}})$ is given in Eq. 3.19 and we have the equivalent posterior PDF (Eq. 3.20. Note that, at this stage, we are not imposing a priori than the stars must be coeval.

The results of the exercise for the M67 open cluster are shown in Fig. 9 where the age PDF has been obtained by marginalising over the other parameters (in this case, distance, reddening, metallicity). Each star yields its PDF in the parameters, and the lower panel shows the full range of PDFs reached by the ensemble of stars in M67. Clearly some stars cannot possibly be members of the cluster, for they have widely discrepant age distributions. This technique allows one to make a further selection (besides proper motions) for true members of the cluster. Note also that some distributions may be affected by some of the underlying assumptions made: 


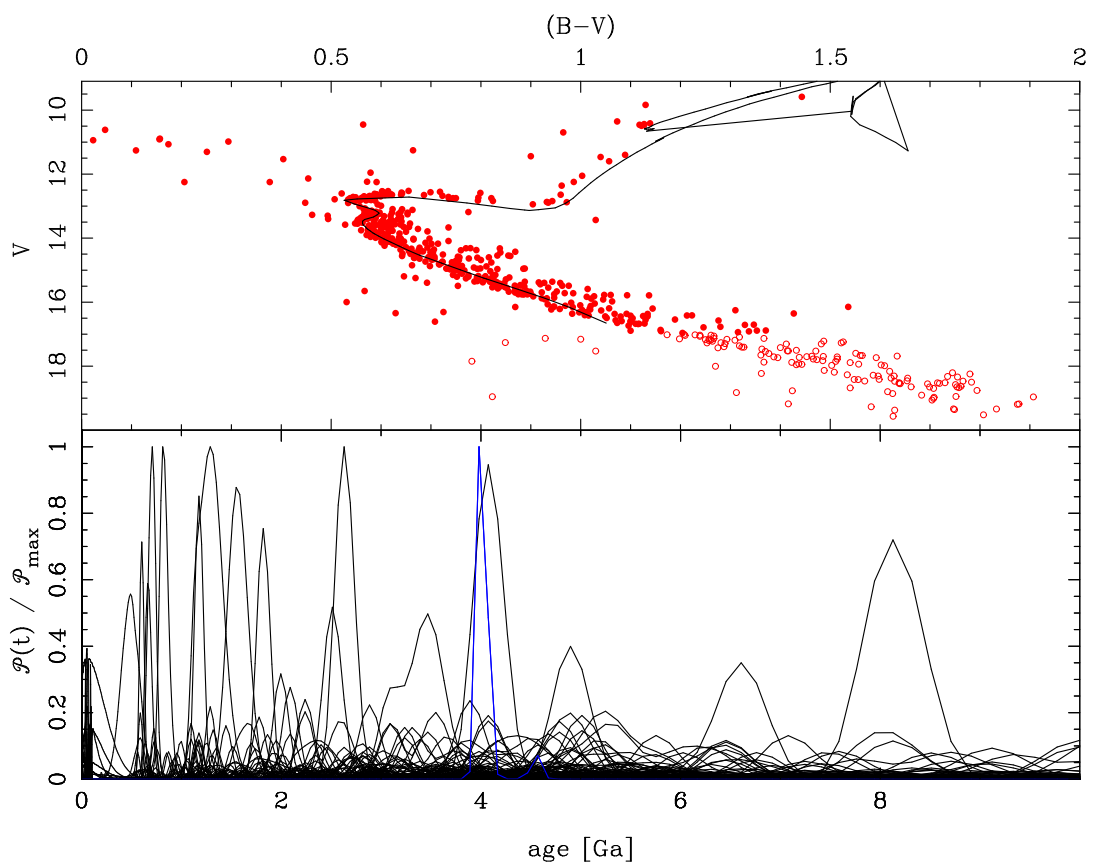

Fig. 9. BayesGM can be used to determine the ages of clusters in the same way as in detached binaries, under the assumption of coevality of its stellar populations, in this case M67 (NGC 2682). Each star produces its age PDF (black lines in the lower panel), whose product yields the cluster's age PDF (blue curve). A prior on metallicity of $[\mathrm{Fe} / \mathrm{H}]=0.0 \pm 0.1$ is assumed, given the spectroscopic measures of both dwarf and giant stars (Santos et al, 2009). Only stars with probabilities of membership larger than 50\% based on proper motions and radial velocities have been used (Yadav et al., 2008). Only stars brighter than $V=17$ are considered, as some sets of tracks appear to present bluer isochrones than observed (Yadav et al., 2008) at fainter magnitudes. The mode of the marginalised PDF on distance modulus is $\mu=9.65$.

some stars are certainly unresolved binaries and clearly the isochrones cannot possibly fit the blue stragglers present which will, nevertheless, produce unsensical PDFs unless they are filtered out.

The product of all the marginalised PDFs yields the PDF of the age of the ensemble as $\pi(t)=\delta\left(t-t_{\star}\right)$ and in this case its mode lies at $3.89 \mathrm{Ga}$, with a sharply-defined PDF. The corresponding isochrone is indicated in the upper panel of Fig. 9. The open circles in the figure are stars fainter than $V=17$ and which appear to be too blue for the set of isochrones (this problem is also found in a different context by An et al. (2007)). Could this be caused by chromospheric 


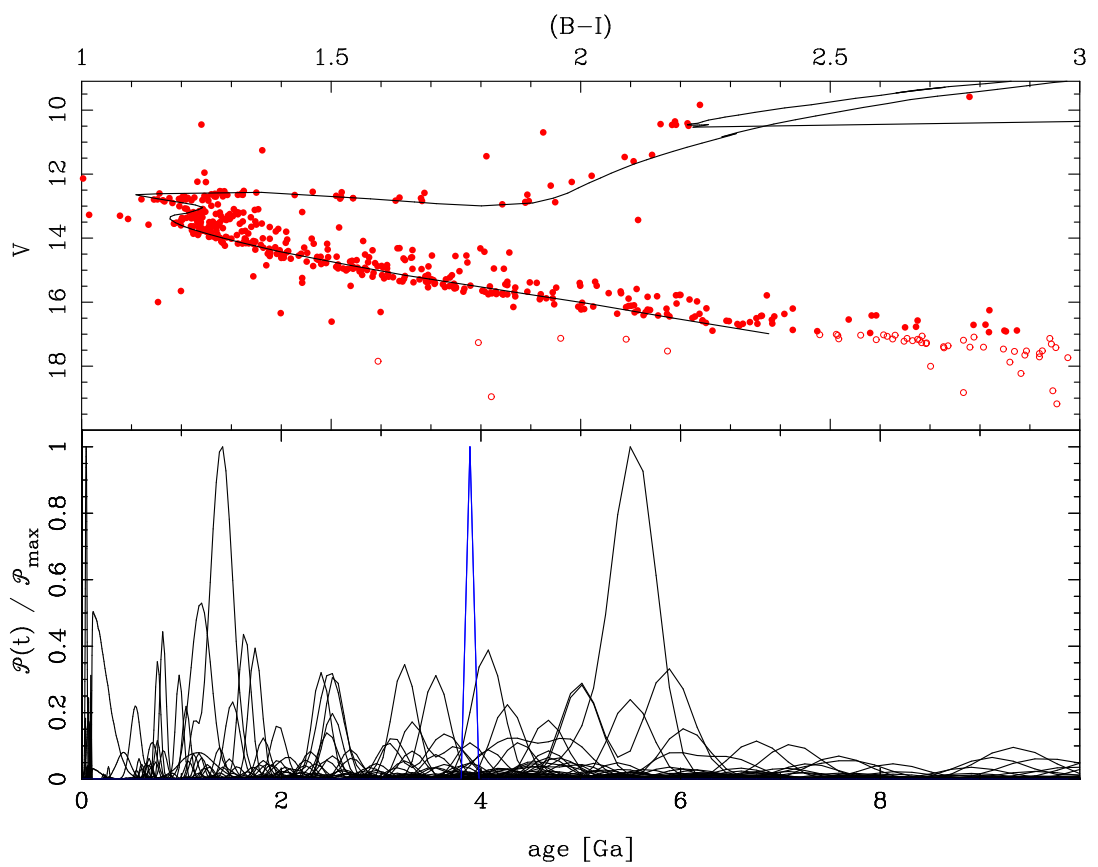

Fig. 10. The same dataset for $M 67$ as in Fig. 9 but this time using the $B, V$ and $I$ photometry yields the same modal age, but a mode of the posterior PDF in distance of $\mu=9.53$. The difference cannot be accounted for extinction and shows the level of systematics at $\delta \mu \sim 0.1$.

activity or is it a problem in the evolutionary tracks?

To check this issue, we can take the set of $(B, V, I)$ independent measures and re-do the analysis. Fig. 10 shows that in this case the mode of the posterior age distribution peaks at $3.98 \mathrm{Ga}$, and the PDF is fully consistent with the one inferred from the $(B, V)$ set. However, the modes of the distance modulus PDFs are significantly different, 9.65 and 9.53 , and cannot be accounted for the uncertainties in the reddening (which was also marginalised out). The outcome of the analysis is that there is a level of systematics that can only be explored using the fully $\mathrm{N}$-dimensional PDFs, to assess correlations between the parameters and possible causes of inconsistencies.

We can now see an example of the multi-dimensional PDFs case by applying BayesGM to globular clusters, with flat priors ${ }^{5}$. Fig. 11 shows the CMD of the old

\footnotetext{
${ }^{5}$ Note however that the set of stellar tracks used only allowed three different values for $[\alpha / \mathrm{Fe}]$, namely $+0.0,+0.2$ and +0.3 .
} 

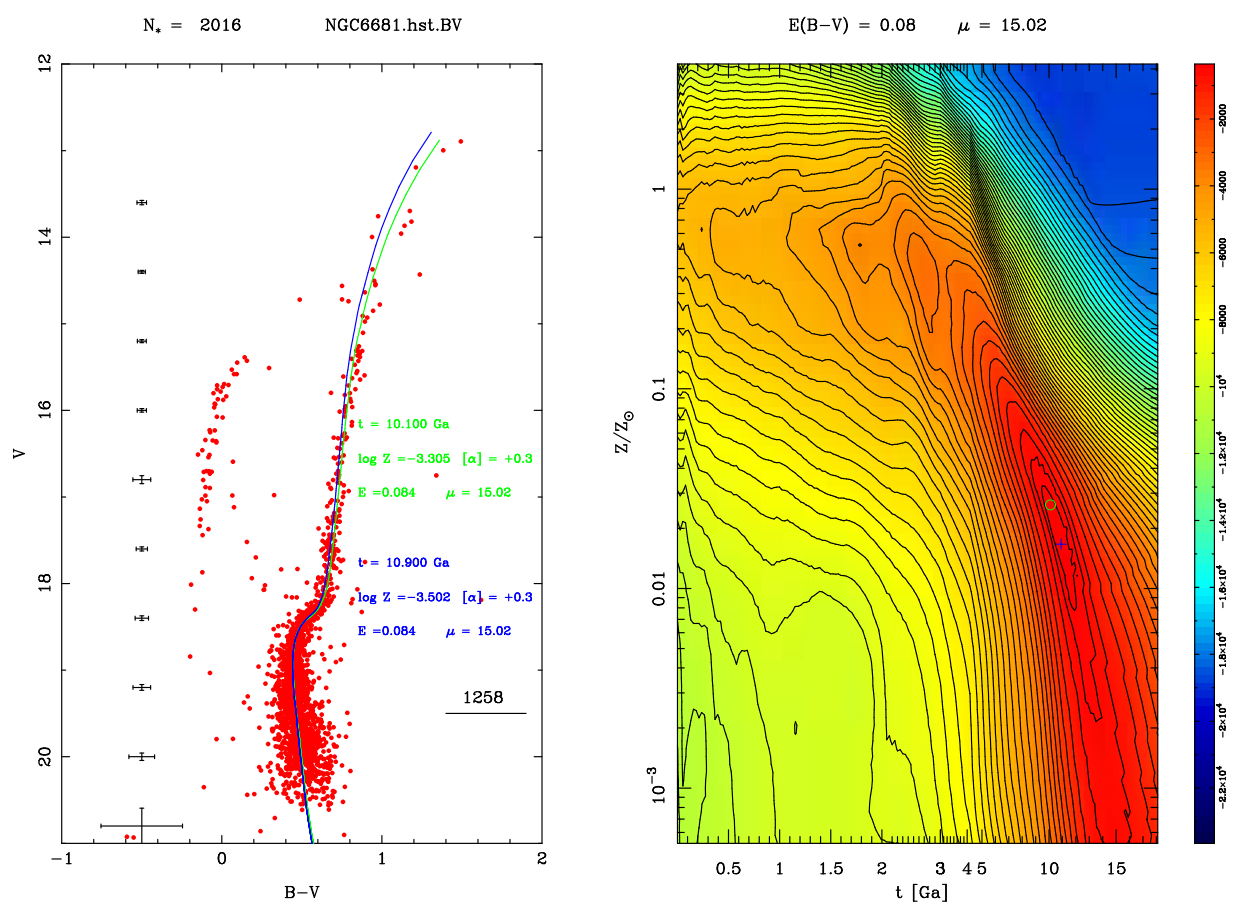

Fig. 11. Lifting the geometrical age-metallicity degeneracy. The left panel shows HST observations of NGC 6681, suitably transformed to the UBVRI system. The typical error bars at each magnitude are indicated on the left. Only the 1258 stars brighter than $V=19.5$ are used. BayesGM yields a posterior PDF on metallicity of $\log Z=-3.502$ with $[\alpha / \mathrm{Fe}]=+0.3$, a colour excess $E(B-V)=0.084$, distance modulus $\mu=15.02$ and age $t=10.9 \mathrm{Ga}$ (blue isochrone, plus sign on the right panel). The solution depends critically on the possible membership of three stars at the top of the RGB. Removing these three stars yields the blue isochrone, while assuming that they are members produces an age which is older by $0.8 \mathrm{Ga}$, the remaining posteriors being the same. In contrast with Fig. 2 where the locus of maximum likelihood followed a long stretch, here the inclusion of the evolutionary speed along each isochrone creates closed contours (right panel), and hence lifts the degeneracy.

globular cluster NGC 6681, observed with HST, assuming the standard transformations to the $B, V$ system. Here the modes of the posterior appear well-defined as well, and the right panel of the Figure shows the PDF marginalised over all parameters but age and metallicity. The probability contours have the same shape as the ones we saw in the age-metallicity degeneracy (Fig. 2), except that this time we can lift entirely the degeneracy: only a tiny area has the maximum probability.

The technique is very powerful, yet subject to some interesting systematics. Fig. 11 also shows the resulting isochrone for the modal age (and metallicity, 
distance, etc) when the top 3 stars are removed. These are very bright stars and one may wonder whether they do belong to the cluster at all. In this case, the modal age shifts by $0.8 \mathrm{Ga}$ to younger ages (still within the top most inner probability contour), and the modal posterior metallicity moves -0.2 dex. This is not, however, a sign of degeneracy because the data set is different, it just shows the sensitivity of some parameters to outliers (the distance and reddening are, quite rightly, unaffected by their presence).

Outliers and possible non members do not always perturb the modes. Fig. 12 shows the ground-based CMD of NGC 6397 and a sharply-peaked marginalised 2dimensional PDF with modes at $16.9 \mathrm{Ga}$ and $Z=-4.2 \mathrm{dex}$. In this case, removing the four brightest stars shifts the modes to $17.5 \mathrm{Ga}$ and $Z=-4.41 \mathrm{dex}$. Taken at face value, this globular cluster appears older than the age of the universe as inferred from a set of completely independent measures (CMB fluctuations and the expansion rate), but in fact points to a systematic in this set of tracks at these very low metallicities ${ }^{6}$.

An important caveat is that an increasingly large number of mainly spectroscopic (but also some photometric) observations is revealing that many globular clusters have multiple, not simple stellar populations. The spread and anticorrelation of $\mathrm{Na}$ and $\mathrm{O}$, for example, may be accounted for in a self-pollution scenario, where the ejecta from the old population lead to a composition of the younger one enriched in $\mathrm{He}, \mathrm{N}, \mathrm{Na}, \mathrm{Al}$, but depleted in $\mathrm{C}, \mathrm{O}$, Ne and $\mathrm{Mg}$. However, the Bayesian technique can, in fact, assign individual probabilities of membership to one or another of the population, precisely because their different abundances may lead to differential evolution and hence positions in the CMDs.

\section{Composite resolved stellar populations}

A variable star formation rate and chemical evolution history give rise to a composite stellar population, which is a mixture of stars of different ages and chemical abundances. Disentangling which populations were formed in this scenario is the main goal of the general inverse problem: Given an observed CMD, what is the distribution of ages of its stellar populations?. This distribution is the basis with which inferences on the star formation and chemical enrichment histories can be made.

The problem is far from trivial, in part due to the apparent age-metallicity degeneracy, and was limited during a long time to a qualitative comparison between the observations and synthetic CMDs with prescribed SFR and $Z(t)$ histories. Aparicio et al. (1990) and Tosi et al. (1991) were the early pioneers to attempt the statistical inversion of the CMDs based on the comparison of number counts in suitably-defined areas of the CMD, and were a step beyond a qualitative analysis. There soon were many other attempts (see, e.g., Gallart, Zoccali \& Aparicio,

\footnotetext{
${ }^{6}$ Since the dataset has been modified as some stars has been excluded, note that the evidence changes, and hence the posteriors must be compared properly normalised.
} 

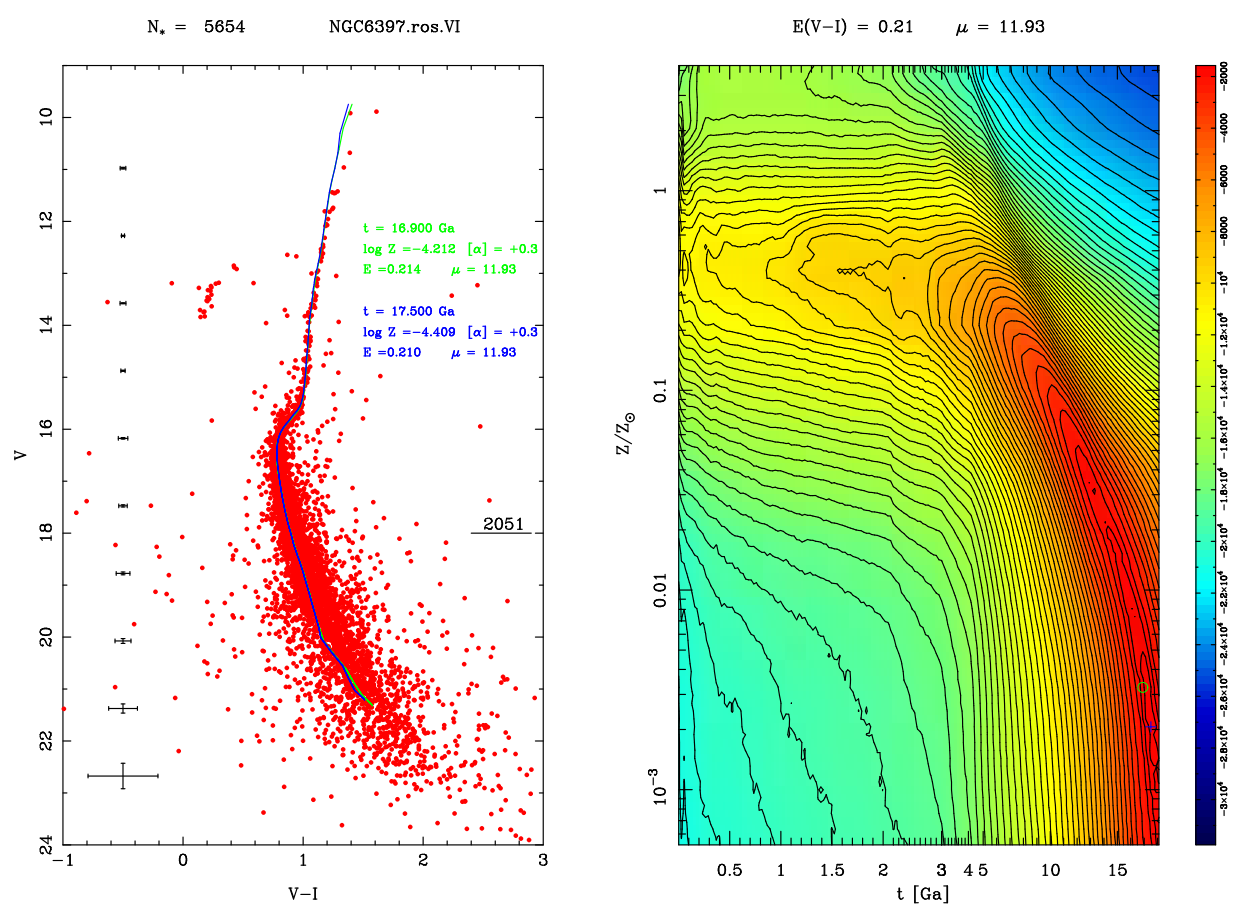

Fig. 12. Ground-based observations of NGC 6397 yield 2051 stars brighter than $V=18$, with average photometric uncertainties as indicated on the left. The age-metallicity degeneracy is lifted (right panel), although in this case the issue of whether the four topmost stars in the RGB belong to the cluster or not does not change the mode of the posterior PDF of the age: in both instances the ages appear to be larger than $16.5 \mathrm{Ga}$, which may point to a limitation in the stellar evolutionary tracks at these low metallicities.

2005, for a review) in this direct approach, including $\mathrm{Ng}$ et al. (2002) who used an optimisation technique with a genetic algorithm. The thorny issue, as we will see, is to compare properly simulated CMDs with observations (Section $\S 8$ ).

Dolphin (1997) (later refined and applied in Dolphin (2002)'s MATCH code at americano.dolphinsim.com/match), Olsen (1999) and Harris \& Zaritsky (2001) (StarFish code www.noao.edu/staff/jharris/SFH) proposed to decompose a generic CMD into a linear combination of 'elemental' CMDs produced by coeval populations of well-defined ages and metallicities. This is also the method use by Makarov \& Makarova (2004) with their StarProbe code. The observed CMD is thus posited to be produced by the linear combination of 'partial' CMDs as

$$
N(i, j)=\sum_{k} R_{k} n(i, j)_{k}
$$

where $N(i, j)$ is the number of stars in the bin $(i, j)$ of the observed CMD, made up of the weighted sum of $k$ partial CMDs with counts $n(i, j)_{k}$ in that bin. If the 
partial CMDs were computed for a nominal $S F R=1 M_{\odot} \mathrm{a}^{-1}$, then the weights $R_{k}$ provide the star formation rate contributed by the $k$-th partial CMD. One can add a foreground CMD to model any underlying contamination, completeness, etc.

Different statistics have been used to infer the values of $R_{k}$ for the set of partial CMDs, although none of them is entirely correct $(c f . \S 8)$. Imposing the non-negativity constraint that $R_{k} \geq 0(\forall k)$ allows one to determine them through iterative or steepest descent methods (in some cases claims of a unique solution have been made, see Dolphin, 2002). For a grid of, say, $10 \times 10$ CMDs spanning 10 ages and 10 metallicities, this is a problem of finding the absolute minimum in a parameter space with $K=100$ dimensions, a non trivial task given the likely presence of many secondary minima. For this reason, the parameter space can be efficiently explored using genetic algorithms to find the absolute maximum corresponding to the best fit ( $\mathrm{Ng}$ et al., 2002; Aparicio \& Hidalgo, 2009), as many secondary minima do exist in this highly dimensional optimisation problem. Their code, IAC-POP, is available at: www.iac.es/galeria/aaj/iac-pop_eng.htm.

Tolstoy \& Saha (1996) pioneered a Bayesian formulation of the problem by pondering on the method to compare datasets drawn from simulations with the actual observed CMD, while Cignoni \& Shore (2006) used the Richardson-Lucy technique to deconvolve the observed CMD in order to produce a 'reconstructed' CMD which can then be compared with simulations.

In fact, the proper way to formulate the problem is to realise that this is, in statistical parlance, an inverse problem which is ill-defined and may have multiple solutions (Craig \& Brown, 1986). Yet claims have been made that unique solutions can nevertheless be found, not only to the functionals defining the star formation and chemical enrichment histories, but also the shape of the IMF, the mass ratio distribution of the unresolved binaries, etc (Vergely et al., 2002; Wilson \& Hurley, 2003). To some extent, this may perhaps be true, but as in all ill-posed this comes at the prize of a compromise between accuracy/smoothness and resolution. To see this, write the probability that a given star with dataset $\overrightarrow{\mathbf{D}}_{i}$ comes from a star formation episode at age $t$ when the star formation rate was $S F R(t)$ and the metallicity $Z(t)$ :

$$
\begin{array}{r}
\mathcal{P}\left[\operatorname{SFR}(t), Z(t) \mid \overrightarrow{\mathbf{D}}_{i}\right]= \\
\pi(t) \pi(Z) \int_{t_{0}}^{t_{1}} \int_{Z_{0}}^{Z_{1}} S F R(t) \mathcal{L}_{\text {phys }}\left(\overrightarrow{\mathbf{D}}_{i} \mid \overrightarrow{\boldsymbol{\vartheta}}, Z(t)\right) d t d Z
\end{array}
$$

where the limits of the integrals come from our prior knowledge $\pi(t)$ that the star must have an age between these limits (could be a least informative prior between 0 to $15 \mathrm{Ga}$ ), and $\pi(Z)$ describes our prior probability on metallicity (could be flat between -5 and 1 , or limited to some range if we have previous measures of $Z$ for these stars). For an ensemble of $N_{\star}$ stars the combined probability that their full CMD arises from the episodes of star formation described by $S F R(t)$ and chemical 
enrichment given by $Z(t)$ becomes

$$
\mathcal{P}_{C M D}[S F R(t), Z(t) \mid \overrightarrow{\mathbf{D}}]=\prod_{i=1}^{N_{\star}} \mathcal{P}\left[S F R(t), Z(t) \mid \overrightarrow{\mathbf{D}}_{i}\right] .
$$

Clearly, given some precision in the photometric data, the fine-grained details of the functions $S F R(t)$ and $Z(t)$ are impossible to constrain, hence the infinity of possible solutions, and the ill-posedness nature of the problem. To regularise the problem one seeks not to maximise this probability, but rather the log of the probability to which one adds a regularisation term which depends on the type of solution one seeks (Craig \& Brown, 1986). The choice varies between terms which penalise variations (i.e., imposes constant solutions to the functions), or terms which penalise gradients or large deviations (i.e., imposes a more or less strict smoothness to the solutions).

The general problem, as far as we are aware, has not yet been solved, and only partial solutions have been explored. For instance, in the case where our prior in metallicity is peaked at some well-defined value $Z_{\star}$ (as is the case in some dwarf galaxies, whose dispersion in metallicity appears to be quite small), one can assume that $\pi(Z)=\delta\left(Z-Z_{\star}\right)$, and the problem reduces to solving for only one function, $S F R(t)$. One could impose a parametric form to this function (say in terms of a piece-wise constant function, or a series of Gaussian bursts) and solve for the parameters of the assumed function. Instead, Hernandez et al. (1999) realised that seeking the maximum of the probability function is equivalent to ask that the variation of the function is zero, ensuring that it is an extremum. In turn, using the Euler-Lagrange equation, $\delta \mathcal{P}_{C M D}\left[S F R(t), Z_{\star}\right]=0$ implies a set of $N_{\star}$ coupled differential equations that can be easily solved. In this way a fully non-parametric solution of $S F R(t)$ was built, with no prior information on shape, form or amplitude. The technique was applied to some nearby dwarf galaxies (Hernandez et al., 2000a), whose HST-based CMDs were well-measured ${ }^{7}$, as well as to the solar neighbourhood probed by Hipparcos (Hernandez et al., 2000b) with a wealth of detail in spite of the few stars used. This was also explored independently by Cignoni et al. (2006) with an entirely different method, and there are many more results in both clusters and nearby galaxies using a variety of methods, most of which are described in recent review articles (Tolstoy, Hill \& Tosi, 2009; Cignoni \& Tosi, 2010).

Interestingly, even if the general problem of determining both functions independently can be solved, we know from chemical evolution theory that there must be some coupling: an enhanced episode of star formation will lead to an enrichment on some time scale which depends on the element and on the details of the nucleosynthetic yields, among other things. Yuk \& Lee (2007) propose to compute in a self-consistent way the chemical enrichment history while inferring at the same time the star formation rate history. However, this is clearly some

\footnotetext{
${ }^{7}$ Dolphin (2002) rightly noted that some zero point offsets which were adopted were wrong, shifting the solutions by a small amount. Much work remains to be done on systematics.
} 
prior information that is imposed, and it is far from clear if, for instance, a closed box model or the assumed recycling are correct. At some point, one can imagine the infall of unenriched gas, or else the accretion of a satellite bringing enriched material. There is clearly ample scope for progress in this field.

\section{Unresolved nopulations and pixel CMDs}

The extreme case of composite populations is reached when their stars cannot be resolved. This is the case, for instance, when analysing the integrated spectra of galaxies or clusters: we only have access to luminosity-weighted estimates of the quantities of interest (age, $Z$, etc). Given the shape of the luminosity function, where a handful of bright stars can dominate the flux of the population (which in fact is dominated in number and mass by the less massive stars), one can easily see the major biases inherent to these techniques.

An intermediate case, which is essential to understand these stellar populations, is the one in the pixels of images of nearby galaxies. In this case, we no longer have to deal with billions of stars (integrated spectra) but rather some $10^{2} \cdots 10^{4}$ stars or so, depending of course on the distance and the size of the pixels. A formalism was proposed Renzini (1998) and observations were pioneered by Bothum (1986) with ground-based observations. Abraham et al. (1999) discussed, in a forward modelling approach, the interpretation of the 4-band images of galaxies in the Hubble Deep field, and the limitations produced by the extinction-colour-metallicity degeneracy. Not limited by the seeing, HST-based studies have dominated the field, which is becoming an essential tool for the understanding of galaxy evolution (e.g Conti et al., 2003; Kassin et al., 2003; Eskridge et al, 2003; Lanyon-Foster et al., 2007; Lee et al., 2011).

While a proper formulation of the inverse problem is still lacking, the forward modelling techniques -which has so far been used-assume a full sampling of the underlyng stellar populations. This may be appropriate for integrated properties, but not in pixels, where the number of stars, while important, is not large enough to ensure the statistical convergence in the properties. This is also the case in the régime where the star formation rate is low, and, in general, in places where the number of stars is small enough as to create stochastic variations in the properties such as luminosities and colours. The importance of these fluctuations is essential also to assess whether the IMF is universal: could the stochastic variations be consistent with samples drawn from the same IMF ?

This stochasticity is equivalent to a lack of convergence in the properties and can be quantified in a simple way, either assuming quasi-Poisson counts (e.g., Cerviño \& Valls-Gabaud, 2003; Cerviño et al., 2002), and through the concept of the lowest luminosity limit, a limit which ensures that statistical fluctuations become unimportant (Cerviño \& Luridiana, 2006; Cerviño \& Valls-Gabaud, 2009). Another approach, more costly in CPU time, is to create Monte Carlo samples. For example Popescu \& Hanson (2009) proposed a code, MASSCLEAN (available at www.physics.uc .edu/ bogdan/massclean.html) to carry out multicolour simulations which do not assume a full sampling of the IMF, and confirm by 
and large the analytical predictions in the quasi-Poisson régime discussed above. This approach is likely to change entirely the interpretation of the integrated properties of stellar clusters (Popescu \& Hanson, 2010a,b; Popescu et al., 2012). In a similar way, new tools have been put forward to produce these stochastic variations in stellar populations. Da Silva et al. (2012) present a code, SLUG (sites.google.com/site/runslug) which is also likely to revisit many results obtained thus far in galaxy evolution.

\section{Best-fit solutions and uncertainties}

An issue which is seldom addressed, if at all, is whether the best-fit solution found (by any method) is also a good fit. There is no guarantee whatsoever that the best straight-line fit to a parabola is a good fit, even if it is the best of all possible within the assumption of a straight line. In some cases the problem is not even tackled. Sometimes a $\chi^{2}$ criterion is used (which is not appropriate, since number counts in any CMD cell or bin follow Poisson statistics), or a comparison with a single model realisation is performed (for instance analysing the residuals in a Hess diagram, which is just a binned version of the CMD, as first used by Hess (1924) in a different context). None of these approaches is satisfactory: one has to deal both with Poisson counts and with the intrinsic variability in the model predictions (as different realisations of the model will invariably yield different results and hence residuals).

Some statistics that have been used in the context of comparing model CMDs (with $\left\{m_{i}\right\}$ stars) with observed CMDs (with $\left\{s_{i}\right\}$ stars) in a selection of $B$ bins or boxes include the following:

1. Pearson's $\chi_{P}^{2}$. This takes into account the variability of the model counts $\left\{m_{i}\right\}$ only:

$$
\chi_{P}^{2}=\sum_{i}^{B} \frac{\left(s_{i}-m_{i}\right)^{2}}{m_{i}} .
$$

2. Modified Neyman's $\chi_{N}^{2}$ which adopts the form

$$
\chi_{N}^{2}=\sum_{i}^{B} \frac{\left(s_{i}-m_{i}\right)^{2}}{\max \left(s_{i}, 1\right)},
$$

but only encapsulates the variability of the observed counts $\left\{s_{i}\right\}$.

3. Dispersion. Kerber et al. (2001) minimise the dispersion defined as

$$
\mathcal{S}^{2}=\sum_{i}^{B}\left(m_{i}-s_{i}\right)^{2} .
$$

While clearly it has the correct behaviour, it does not take into account the Poisson distribution of both model and observed counts. Could the minimum 
$\mathcal{S}^{2}$ value reached be different should a different realisation of the model $\left\{m_{i}\right\}$ be used for the comparison?

4. Percentile position. Kerber et al. (2001) also use the percentile position of $\left|s_{i}-m_{i}\right|$ within the distribution of $\left|m_{i}=m_{i j}\right|$ with $j=1, \cdots N_{\text {sim }}$ and $m_{i j}$ being Poisson realisations with parameter $m_{i}$ in each bin $i$. Then the statistic

$$
\mathrm{pss}=\sum_{i}^{B}\left(1-p_{i}\right)
$$

is minimised. This tackles the part of the intrinsic variability of the model outcomes, but not the one from the observations.

5. $\chi^{2}$ statistic for Poisson variables. Both Ng (1998) and Mighell (1999) point out that the number counts in the bins the CMD has been divided into follow Poisson statistics, not Gaussian ones (unless we are in the large numbers limit, which will not be the case in sparsely-populated bins). The statistic proposed is

$$
\chi_{\gamma}^{2}=2 \sum_{i} \frac{\left[s_{i}+\min \left(n_{i}, 1\right)-m_{i}\right]^{2}}{s_{i}+1},
$$

and many codes have implemented this to assess the goodness (or otherwise) of their fits. However, comparing Poisson counts is tricky, as all high energy physicits know. While the sum of Poisson variates is also Poisson distributed (see, e.g., Cerviño \& Valls-Gabaud, 2003, for some consequences in the context of stellar populations), the difference of Poisson counts, such as $m_{i}-s_{i}$ which one could naively use, do not follow Poisson statistics but are distributed following a Skellam distribution (the fact that the variate may become negative is a clear hint).

6. Poisson likelihood ratio. Dolphin (2002) correctly argued that the proper analogy with the $\chi^{2}$ ratio (which only applies to Gaussian statistics) for Poisson variates is the ratio

$$
-2 \ln \text { PLR }=2 \sum_{i}\left(m_{i}-s_{i}+s_{i} \ln \left(\frac{s_{i}}{m_{i}}\right)\right),
$$

as proposed by Baker \& Cousins (1983). However, using a likelihood ratio has some constraints that makes this quantity unsuitable for seeking a proper comparison (see below).

The likelihood of a model is obviously a relative probability. If we have two models $A$ and $B$ giving likelihoods $\mathcal{L}_{\mathcal{A}}$ and $\mathcal{L}_{\mathcal{B}}$, model $A$ is $\mathcal{L}_{\mathcal{A}} / \mathcal{L}_{\mathcal{B}}$ times as likely as model $B$. This is also known as the bookmakers' odds (Syer \& Saha, 1994). To compare two likelihoods or use a likelihood ratio, however, two conditions are essential (Protassov et al., 2002), but are too often overlooked: 
(i) The models must be nested.

(ii) The values of the parameters must not reach zero.

The first condition implies that, for example, one cannot use a likelihood ratio for comparing a fit using a polynomial and another one using an exponential function. It can be used to compare the fits obtained by a polynomial of degree $k$ with another fit using a polynomial of degree $j \neq k$. The second condition, of strict positivity, implies that one cannot use a likelihood ratio when decomposing a CMD into a linear combination of partial CMDs, as quite a few odd partial CMDs are unlikely to contribute at all (say young populations to a globular cluster). Necessarily some $R_{k}=0$ will happen in Eq. 6.1 and the likelihood ratio cannot be applied.

At any rate, $\chi^{2}$-like statistics such as the ones above depend on the size of the bins the CMD is divided into, and care must be taken when assessing their significance in comparison with CMDs with different cell/bin sizes. In addition, one has to take into account the intrinsic variability of the model predictions, so a comparison between a single realisation and an observed CMD makes very little sense. Obviously one can only take into account a Poisson dispersion in the observed counts, but models have not only this intrinsic variability but also one associated with them, even for a fixed set of values for the parameters.

A nearly size-independent statistic was suggested by Bell et al. (2008) where the rms deviation of the data with respect to the model is minimised, and takes explicitely into account the Poisson variability of the model. For a set of $B$ bins, one forms the distribution of

$$
\sigma / \text { total }=\sqrt{<\sigma^{2}>}\left[\frac{1}{B} \sum_{i}^{B} s_{i}\right]^{-1}
$$

where

$$
<\sigma^{2}>=\frac{1}{B}\left[\sum_{i}^{B}\left(s_{i}-m_{i}\right)^{2}-\sum_{i}^{B}\left(m_{i}^{\prime}-m_{i}\right)^{2}\right] .
$$

This statistic takes into account the variability of the model, as $\left\{m_{i}^{\prime}\right\}$ are Poisson realisations of the model with expectation values $\left\{m_{i}\right\}$, but not the uncertainties in the observed counts $\left\{s_{i}\right\}$. It is, in fact, designed to detect the fluctuations in the observed number counts with respect to the models.

Should we know the distribution function of the model, we could apply the standard statistical tools to infer the proper credibility intervals. This is obviously not the case in CMDs, but we can generate samples from the model (a sample of infinite size would be as good as the model). Different samples (different realisations) are likely to populate regions of the CMD which may not coincide with the observed ones, we hence need to smooth out the model and data for a proper comparison. The simplest way of doing this is binning, with the underlying assumption that the model distribution function is constant within the bin (hence 
small bins are preferred, but they should be large enough that bins contain both model and observed stars as much as possible).

If we divide the CMD into $B$ cells, of arbitrary sizes and shapes, each containing $\left\{s_{i}\right\}$ observed stars (with a total of $S=\sum_{i}^{B} s_{i}$ stars), and $\left\{m_{i}\right\}$ model stars (with a total of $M=\sum_{i}^{B} m_{i}$ stars), each cell has a probability $w_{i}$ of having the appropriate number of stars (model or observed), constant within the bin. The probability distribution function for the bin occupancies, multi-variate distribution of counts, will be given by a multinomial distribution

$$
P\left(s_{i}, m_{i} \mid w_{i}\right)=M ! S ! \prod_{i=1}^{B} \frac{w_{i}^{m_{i}+s_{i}}}{m_{i} ! s_{i} !},
$$

where the weigths $\left\{w_{i}\right\}$ of the distribution function are unknown. We only have the constraint, given by the normalisation, that $\sum_{i}^{B} w_{i}=1$. We can treat the weights as nuisance parameters and marginalise over them using the identity

$$
\left(\prod_{i=1}^{B} \int w_{i}^{n_{i}} d w_{i}\right) \delta\left(\sum w_{j}-1\right)=\frac{1}{(N+B-1) !} \prod_{i=1}^{B} n_{i} !,
$$

and we get

$$
P\left(s_{i}, m_{i}\right)=\frac{M ! S !(B-1) !}{(M+S+B-1) !} \prod_{i=1}^{B} \frac{\left(m_{i}+s_{i}\right) !}{m_{i} ! s_{i} !},
$$

which is equal to $P\left(s_{i} \mid m_{i}\right) P\left(m_{i}\right)$. And likewise with $P\left(m_{i}\right)$, and so Bayes' theorem gives us

$$
P\left(s_{i} \mid m_{i}\right)=\frac{S !(M+B-1) !}{(M+S+B-1) !} \prod_{i=1}^{B} \frac{\left(m_{i}+s_{i}\right) !}{m_{i} ! s_{i} !} ; .
$$

For a fixed number of bins $B$, model stars $M$ and observed stars $S$, the first term is a constant and hence

$$
\text { Prob } \propto W=\prod_{i=1}^{B} \frac{\left(m_{i}+s_{i}\right) !}{m_{i} ! s_{i} !} .
$$

This statistic was first proposed by Saha (1998) and has been widely used, for example in comparisons of N-body simulations with discrete data sets (Sevenster et al., 1999; Beaulieu et al., 2000) or indeed in inversions of CMDs (Hernandez et al., 2000a,b; Kerber et al., 2002), correlations between the SFR history and the glaciation epochs (De La Fuente Marcos \& De La Fuente Marcos, 2004), and in setting constraints on the properties of clusters (e.g., Rengel et al., 2002; Kerber \& Santiago, 2005). Contrary to some baseless claims (Dolphin, 2002), it does allow a proper comparison between data and models. Note that it takes model counts 
and observed counts on the same footing. If $M$ is arbitrarily large, the bins can be made small enough as to contain one observed star at most, so that the probability goes as $\prod_{k}\left(1+m_{k}\right)$ where $k$ is the running index for boxes with $s_{k}=1$, and with $m_{k} \gg 1$ we get the same result as in the continuous distribution case.

More properly in the context of multidimensional Poisson counts, if $M$ and $S$ are not fixed but are the expectations values of the totals when $s_{i}$ and $m_{i}$ are drawn from a Poisson process, we get a modified $W$ statistic (Saha, 2003) as

$$
P\left(s_{i} \mid m_{i}\right)=\frac{e^{-M} M^{M} e^{-S} S^{S}(B-1) !}{(M+S+B-1) !} \prod_{i=1}^{B} \frac{\left(m_{i}+s_{i}\right) !}{m_{i} ! s_{i} !}
$$

It is clear that, normalisations aside (which can be fixed if indeed $B, M$ and $S$ are kept fixed), Saha's $W$ statistic (Eq. 8.14) can be used to perform either

- Parameter fitting: Just compute the distribution of $W$ for a given data set and different model parameters. That is, fix $\left\{s_{i}\right\}$, vary model parameters (hence $\left\{m_{i}\right\}$ ) then read off parameter estimates and confidence intervals for the parameters.

- Goodness of fit: Here we want the distribution of $W$ for fixed model parameters and various simulated data sets. Fix model parameters (so $\left\{m_{i}\right\}$ are fixed), then vary simulated $\left\{s_{i}=m_{i}^{\prime}\right\}$. Then compare the distribution of $W$ with the distribution obtained from the actual data. The extent to which both distributions (actually, samples of $W$ ) can be drawn from the same underlying (and unknown) distribution function gives a proper measure of the goodness of the fit.

It is therefore the statistic of choice to be used in the context of CMD modeling, and turns out to be far superior to the classical Mann-Withney test for 2 samples.

In summary, the determination of stellar ages using stellar evolutionary theory has a proper Bayesian formalism, which allows one to infer not a single value (which makes little sense from a statistical point of view) but rather the posterior distribution function which can be used to constrain both the properties of the stars and the predictions of the models. It is beyond the scope of this chapter to analyse the comparison between different sets of tracks and different assumptions on the systematics. This ought to be tackled, in fact, by a double blind experiment in which a referee generates a set of simulated CMDs from a given (but unknown to the referee) set of isochrones, including realistic photometric errors, which are then analysed by different teams using different isochrones and assumptions. Only in this way the actual distribution function of ages can be quantitatively estimated, and the robust confirgurations which give a narrow distribution, independently of sets and assumptions, identified.

\section{Acknowledgements}

The author would like to thank the "Formation permanente du CNRS" for financial support. This research has made use of NASA's Astrophysics Data System. 


\section{References}

Abraham, R.G. et al. 1999, MNRAS, 303, 641

An, D. et al. 2007, ApJ, 655, 233

Aparicio, A. et al. 1990, A\&A, 240, 262

Aparicio, A., Hidalgo, S.L. 2009, AJ, 138, 558

Bailer-Jones, C.A.L. 2011, MNRAS, 411, 435

Baker, S., Cousins, R.D. 1983, Nucl. Inst. Meth. Phys. Res., 221, 437

Bayes, T. 1763, Phil. Trans. Roy. Soc. 53, 370; 54, 296

Beaulieu, S., et al. 2000, AJ, 120, 855

Becker, S.A., Mathews G.J. 1983, ApJ, 270, 155

Bell, E.F. et al. 2008, ApJ, 680, 295,

Breddels, M.A. et al. 2010, A\&A, 511, 16

Brott I., et al. 2011, A\&A, 530, A115

Bruntt, H., Southworth, J. 2008, J. Phys. Conf. Series, 118, 012012

Bothum, G.D. 1986, AJ, 91, 507

Burnett, B., Binney, J. 2010, MNRAS, 407, 339

Casagrande, L. et al. 2010, A\&A, 512, A54

Casagrande, L. et al. 2011, A\&A, 530, A138

Cerviño, M., Luridiana, V. 2006, A\&A, 451, 475

Cerviño, M., Valls-Gabaud, D. 2003, MNRAS, 338, 481

Cerviño, M., Valls-Gabaud, D. 2009, Ap\&SS, 324, 91

Cerviño, M. et al. 2002, A\&A, 381, 51

Chanamé, J., Ramírez, I. 2012, ApJ, 746, 102

Charlier, C.V.L. 1889, Publ. Astronomischen Gesell., 19, 1

Cignoni, M., Degl'Innocenti, S., Prada Moroni, P.G., Shore, S.N. 2006, A\&A, 459, 783

Cignoni, M., Shore, S.N. 2006, A\&A, 454, 511

Cignoni, M., Tosi, M. 2010, Adv. Ast., 2010, 158568 
Collins, G.W., Sonneborn, G.H. 1977, ApJS, 34, 41

Collins, G.W., Smith, R.C. 1985, MNRAS, 213, 519

Conti, A., et al. 2003, AJ, 126, 2330

Cousin, R. 1995, Am. J. Phys, 63, 398

Craig, I.J.D., Brown, J.C. 1986, Inverse problems in Astronomy (Bristol: Adam Hilger)

Dale, A.I. 1982, Arch. Hist. Exact Sci., 27, 23

Da Silva, L. et al. 2006, A\&A, 468, 609

Da Silva, R.L., Fumagalli, M., Krumholz, M. 2012, ApJ, 645, 145

De La Fuente Marcos, R., De La Fuente Marcos, C. 2004, New Ast., 10, 53

DeVorkin, D.H. 2000, Henry Norris Russell, Dean of American Astronomers (Princeton: Princeton University Press)

Dolphin, A. 1997, New Ast., 2, 397

Dolphin, A. 2002, MNRAS, 332, 91

Dose, V. 2003, Rep. Prog. Phys., 66, 1421.

Duerbeck, H.W. 2006, in Organizations and Strategies in Astronomy, Vol. 7, ed. A. Heck (Berlin: Springer Verlag), 383

Eskridge, P.B. et al. 2003, ApJ, 586, 923

Flannery, B.P., Johnson, B.C. 1982, ApJ, 263, 166

Gallart, C., Zoccali, M., Aparicio, A. 2005, ARA\&A, 43, 387

Gennaro, M., Prada Moroni, P.G., Tognelli, E. 2012, MNRAS, 420, 986

Gleissberg, W. 1940, Pub. Istanbul Obs., 13, 2

Gregory, P. 2005, Bayesian logical data analysis for the physical sciences (Cambridge: Cambridge University Press)

Harmanec, P., Prs̆a, A. 2011, Pub. Ast. Soc. Aus., 123, 976

Harris, J., Zaritsky, D. 2001, ApJS, 136, 25

Hearnshaw, J.B. 1986, The analysis of starlight. One hundred and fifty years of astronomical spectroscopy (Cambridge: Cambridge University Press)

Haffner, H., Heckmann, O. 1937, Veröff Univ. Sternwarte Göttingen, 55, 77 
Hermann, D.B. 1994, Ejnar Hertzsprung: Pionier der Sternforschung (Berlin: Springer-Verlag)

Hernandez, X., Valls-Gabaud, D. 2008, MNRAS, 383, 1603

Hernandez, X., Valls-Gabaud, D., Gilmore, G. 1999, MNRAS, 304, 705

Hernandez, X., Gilmore, G., Valls-Gabaud, D. 2000a, MNRAS, 317, 831

Hernandez, X., Valls-Gabaud, D., Gilmore, G. 2000b, MNRAS, 316, 605

Hertzsprung, E. 1911, Publ. Astrophys. Obs. Potsdam, No. 63

Hess, R. 1924, Probleme der Astronomie. Festschrift fur Hugo v. Seeliger (Berlin: Springer Verlag), p. 265

Hillenbrand, L.A., White, R.J. 2004, ApJ, 604, 741

Hobson, M.P. et al. 2010, Bayesian methods in cosmology (Cambridge: Cambridge University Press)

Hurley, J., Tout, C.A. 1989, MNRAS, 300, 977

Javiel, S.C., Santiago, B.X., Kerber, L.O. 2005, A\&A, 431, 73

Jeffery, E.J., von Hippel, T., Jefferys, W.H., Winget, D.E., Stein N., De Gennaro, S. 2007, ApJ, 658, 391

Jeffery, E.J., von Hippel, T., De Gennaro, S., van Dyk, D.A., Stein N., Jefferys, W.H. 2011, ApJ, 730, 35

Jørgensen, B.R., Lindegren, L. 2005, A\&A, 436, 127

Kassin, S.A. et al. 2003, AJ, 126, 1276

Kerber, L.O., Javiel, S.C., Santiago, B.X. 2001, A\&A, 365, 424

Kerber, L.O. et al. 2002, A\&A, 390, 121

Kerber, L.O., Santiago, B.X. 2005, A\&A, 435, 77

Kerber, L.O., Girardi, L., Rubele, S., Cioni, M.-R. 2009, A\&A, 499, 697

Lachaume, R., Dominik, C., Lanz, T., Habing, H.J. 1999, A\&A, 348, 897

Lanyon-Foster, M.M., Conselice, C.J., Merrifield, M.R. 2007, MNRAS, 380, 571

Laplace, P.S. 1812, Théorie analytique des probabilités (Paris: Courcier)

Lastennet, E., Valls-Gabaud, D. 1996, in The origins, evolutions and desitinies of binary stars in clusters, Astonomical Society of the Pacific Conference Series, Vol. 90, E.F. Milone and J.C. Mermilliod (eds), 464 
Lastennet, E., Valls-Gabaud, D. 2002, A\&A, 396, 551

Lastennet, E. et al. 2003, A\&A, 409, 611

Lastennet, E. et al. 1999, A\&A, 349, 485

Lebreton, Y. 2000, ARA\&A, 38, 35

Lee, J.H. et al. 2011, ApJ, 740, 42

Ludwig, H.-G. et al. 1999, A\&A, 346, 111

Luri, X., Torras, J., Figueras, F. 1992, A\&A, 259, 382

Maeder, A., Peytremann, E. 1970, A\&A, 7, 120

Maeder, A., Meynet, G. 2012, Rev. Mod. Physics, 84, 25

Maeder, A. 1974, A\&A, 32, 177

Makarov, D.I., Makarova, L.N. 2004, Astrophysics, 47, 229

Malkov, O.Y. et al. 2010, MNRAS, 401, 695

Mathieu, R.D., Baraffe, I., Simon, M., Stassun, K.G., \& White, R. 2007, in Protostars and Planets $V$ (Tucson: University of Arizona Press), 411

Mayne, N.J., Naylor, T. 2008, MNRAS, 386, 261

Meissner, F., Weiss, A. 2005, A\&A, 456, 1085

Meyer-Hofmeister, E. 1969, A\&A, 2, 143

Mighell, K.J. 1999, ApJ, 518, 380

Monteiro, H., Dias, W.S., Caetano, T.C. 2010, A\&A, 516, A2

Naylor, T., Jeffries, R.D. 2006, MNRAS, 373, 125

Naylor, T. 2009, MNRAS, 399, 432

Ng, Y.K. 1998, A\&AS, 132, 133

Ng, Y.K., Brogt E., Chiosi, C., Bertelli, G. 2002, A\&A, 392, 1129

Nielsen, A.V. 1969, Centaurus, 9, 219

Nordström, B. et al. 2004, A\&A, 418, 989

Olsen, K.A.G. 1999, AJ, 117, 2244

Patenaude, M. 1978, A\&A, 66, 225 
Perrin, M.-N., Cayrel de Strobel, G., Cayrel, R., Hejlesen, P.M. 1977, A\&A, 54, 779

Protassov, R., van Dyk, D.A., Connors, A., Kashyap, V.L., Siemiginowska, A. 2002, ApJ, 571, 545

Pont, F., Eyer, L. 2004, MNRAS, 351, 487

Popescu, B., Hanson, M.M. 2009, AJ, 138, 1724

Popescu, B., Hanson, M.M. 2010a, ApJ Letters, 713, L21

Popescu, B., Hanson, M.M. 2010b, ApJ, 724, 296

Popescu, B., Hanson, M.M., Elmegreen, B.G. 2012, ApJ, 751, 122

Ramírez, I., Meléndez, J. 2005, ApJ, 626, 465

Reddy, B.E., Tomkin, J., Lambert, D.L., Allende Prieto, C. 2003, MNRAS, 340, 304

Rengel, M., Mateu, J., Bruzual, G. 2002, in Extragalactic star clusters, IAU Symp. 207, D. Geisler, E.K. Grebel and D. Minitti (eds), 716

Renzini, A. 1998, AJ, 115, 2459

Renzini, A., Buzzoni A. 1983, Mem. Soc. Ast. Ital., 54, 739

Robertson, J.W., 1974, ApJ, 191, 67

Rosenberg, H. 1910, Ast. Nach., 186, 71

Rosenberg, H. 1929, in Handbuch der Astrophysik, Vol. 2, Grundlagen der Astrophysik, p. 380

Rosenberg, H. 1936, ApJ, 83, 67

Russell, H.N. 1912, Proc. Phil. Soc. Amer., 51, 569

Russell, H.N. 1914a, Popular Ast., 22, 275

Russell, H.N. 1914b, Popular Ast., 22, 331

Russell, H.N. 1931, MNRAS, 91, 951

Russell H.N., Dugan R.S. \& Stewart J.Q., 1927, Astronomy, Vol. II (Boston: Ginn and Co.)

Saha, P. 1998, AJ, 115, 1206

Saha, P. 2003, Principles of data analysis (London: Cappella Archive) 
Salaris, M., Cassisi, S. 2005, Evolution of stars and stellar populations (Chichester: John Wiley \& Sons)

Santos, N.C., et al. 2009, A\&A, 493, 309

Schaltenbrand, R.A. 1974, A\&AS, 18, 27

Sevenster, M. et al. 1999, MNRAS, 307, 584

Shapley, H. (ed), 1960, Source book in Astronomy 1900-1950 (Cambridge: Harvard University Press), p. 247

Siess, L., Forestini, M., Dougados, C. 1997, A\&A, 324, 556

Simon, M. 2008, in The Power of Optical/IR Interferometry: Recent Scientific Results and 2nd Generation, A. Richichi, F. Delplancke, F. Paresce, \& A. Chelli (eds) (Berlin: Springer), 227

Smith, R.W. 1977, Dudley Observatory Reports, 13, 9

Soderblom, D.R. 2010, ARA\&A, 48, 581

Southworth, J. 2011, MNRAS, 417, 2166

Stello, D. et al. 2009, ApJ, 700, 1589

Strand, K. AA. 1968, Pub. Ast. Soc. Pacific, 80, 51

Syer, D., Saha, P. 1994, ApJ, 427, 714

Takeda, G., Ford, E.B., Sills, A., Rasio, F.A., Fischer, D.A., Valenti, J.A. 2007, ApJS, 168, 297

Tolstoy, E., Saha, A. 1996, ApJ, 462, 672

Tolstoy, E., Hill, V., Tosi, M. 2009, ARA\&A, 47, 371

Torres, G. 2010, AJ, 140, 1158

Torres, G., Andersen, J., Giménez, A. 2010, Ast. Astrophys. Rev., 18, 67

Tosi, M., Greggio, L., Marconi, G., Focardi, P. 1991, AJ, 102, 951

Trotta, R. 2008, Contemporary Physics, 49, 71

Valenti, J.A., Fischer, D.A. 2005, ApJS, 159, 141

VandenBerg, D.A., Bergbusch, P.A., Dowler, P.D. 2006, ApJS, 162, 375

VandenBerg, D.A., Casagrande, L., Stetson ,P.B. 2010, AJ, 140, 1020

van Dyk, D.A. et al. 2009, Ann. Appl. Stat., 3, 117 
Vergely, J.-L., Köppen, J., Egret, D., Bienaymé, O. 2002, A\&A, 390, 917

Vogt, H. 1926, Ast. Nach., 226, 301

von Hippel, T. 2005, ApJ, 622, 565

von Hippel, T. et al. 2006, ApJ, 645, 1436

Waterfield, R.L. 1956, Jour. Brit. Ast. Assoc., 67, 1

Wilson, R.E., Hurley, J.R. 2003, MNRAS, 344, 1175

Yadav, R.K.S. et al. 2008, A\&A, 484, 609

Yıldız, M. 2007, MNRAS, 374, 1264

Yildız, M. et al. 2006, MNRAS, 368, 1941

Young, P.A., Mamajek, E.E., Arnett, D., Liebert, J. 2001, ApJ, 556, 230

Yuk, I.-S., Lee, M.G. 2007, ApJ, 668, 876

Zwitter, T. et al. 2010, A\&A, 552, A54

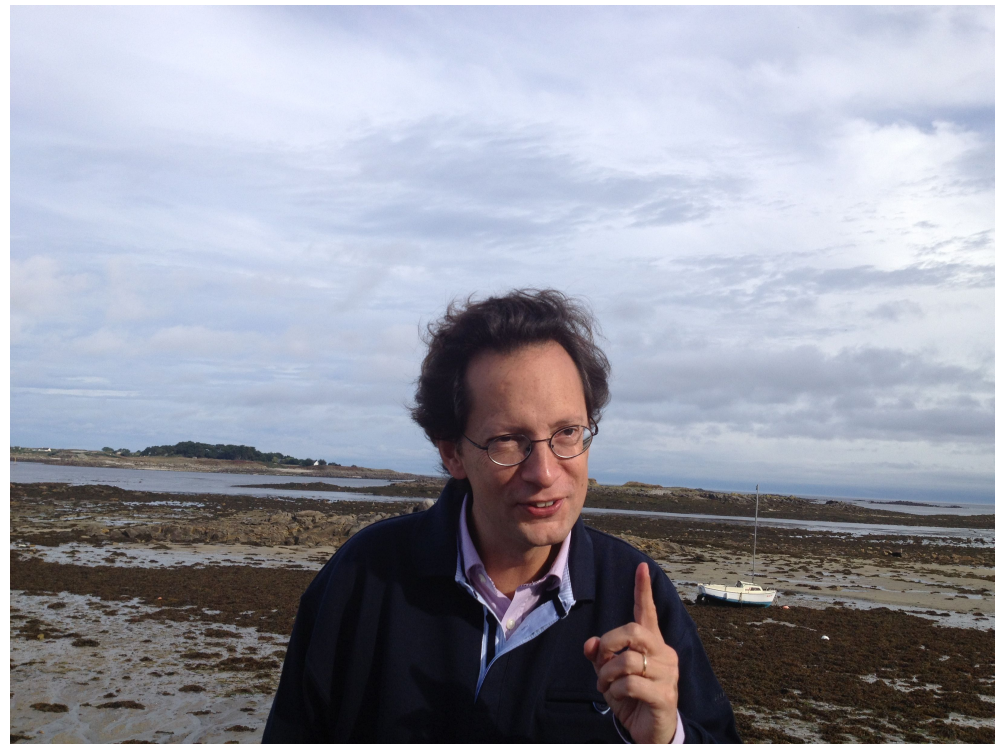

